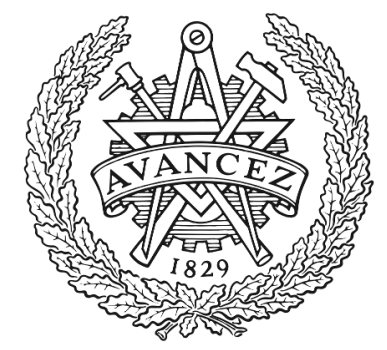

CHALMERS

UNIVERSITY OF TECHNOLOGY

\title{
General method for atomistic spin-lattice dynamics with first-principles accuracy
}

Downloaded from: https://research.chalmers.se, 2023-04-26 13:15 UTC

Citation for the original published paper (version of record):

Hellsvik, J., Thonig, D., Modin, K. et al (2019). General method for atomistic spin-lattice dynamics with first-principles accuracy. PHYSICAL REVIEW B, 99(10).

http://dx.doi.org/10.1103/PhysRevB.99.104302

N.B. When citing this work, cite the original published paper. 


\title{
General method for atomistic spin-lattice dynamics with first-principles accuracy
}

\author{
Johan Hellsvik,,${ }^{1,2, *}$ Danny Thonig, ${ }^{3}$ Klas Modin,,${ }^{4,5}$ Diana Iuşan, ${ }^{3}$ Anders Bergman, ${ }^{3}$ Olle Eriksson, ${ }^{3,6}$ \\ Lars Bergqvist, ${ }^{7,8}$ and Anna Delin ${ }^{7,8,3, \dagger}$ \\ ${ }^{1}$ Nordita, Roslagstullsbacken 23, SE-106 91 Stockholm, Sweden \\ ${ }^{2}$ Department of Physics, KTH Royal Institute of Technology, SE-106 91 Stockholm, Sweden \\ ${ }^{3}$ Department of Physics and Astronomy, Uppsala University, Box 516, SE-75120 Uppsala, Sweden \\ ${ }^{4}$ Department of Mathematics, Chalmers University of Technology, Gothenburg, Sweden \\ ${ }^{5}$ Department of Mathematics, University of Gothenburg, Gothenburg, Sweden \\ ${ }^{6}$ School of Science and Technology, Örebro University, SE-701 82 Örebro, Sweden \\ ${ }^{7}$ Department of Applied Physics, School of Engineering Sciences, KTH Royal Institute of Technology, Electrum 229, SE-16440 Kista, Sweden \\ ${ }^{8}$ SeRC (Swedish e-Science Research Center), KTH Royal Institute of Technology, SE-10044 Stockholm, Sweden
}

(Received 6 July 2018; revised manuscript received 27 November 2018; published 15 March 2019)

\begin{abstract}
We present a computationally efficient and general first-principles based method for spin-lattice simulations for solids and clusters. The method is based on a coupling of atomistic spin dynamics and molecular dynamics simulations, expressed through a spin-lattice Hamiltonian, where the bilinear magnetic term is expanded up to second order in displacement. The effect of first-order spin-lattice coupling on the magnon and phonon dispersion in bcc Fe is reported as an example, and we observe good agreement with previous simulations. We also illustrate the coupled spin-lattice dynamics method on a more conceptual level, by exploring dissipation-free spin and lattice motion of small magnetic clusters (a dimer, trimer, and tetramer). The method discussed here opens the door for a quantitative description and understanding of the microscopic origin of many fundamental phenomena of contemporary interest, such as ultrafast demagnetization, magnetocalorics, and spincaloritronics.
\end{abstract}

DOI: 10.1103/PhysRevB.99.104302

\section{INTRODUCTION}

The way in which atoms vibrate around their equilibrium positions as a function of temperature is of fundamental importance for a range of physical properties of solids, for example, thermal expansion, specific heat, thermal conductivity, and superconductivity. These vibrations can be studied computationally using molecular dynamics (MD) simulations, today a mature and widely used technique in computational materials science. Phonon spectra and other properties related to the atomic vibrations are presently routinely computed. To address systems with millions of atoms with MD, empirical potentials are usually necessary. Only for relatively small systems are MD simulations at the first-principles level feasible $[1,2]$.

In systems with magnetic order, there also exist collective motion of the spins, in addition to the above-mentioned lattice vibrations. The standard approach to simulate the time evolution of the spin texture is to propagate the Landau-LifshitzGilbert (LLG) equation. Both continuum models (usually called "micromagnetics") [3] and atomistic models, so-called atomistic spin dynamics (ASD) [4-8], have been developed. In principle, spin motion can also be addressed directly at the first-principles level [4,9-11]. This can be achieved in the framework of time-dependent density functional theory, [12] although this normally requires too much computing resources and time to be realistic. Also, in order to take dissipation and fluctuations into account in spin-dynamic sim-

\footnotetext{
*hellsvik@kth.se

†annadel@kth.se
}

ulations, a phenomenological stochastic approach is typically employed, for details see, e.g., Ref. [8]. A full microscopic description of dissipation would require explicit descriptions of all the spin-electron couplings as well as all spin-lattice couplings.

In reality, the atomic magnetic moment and lattice degrees of freedom are always more or less coupled, a coupling that is mediated by the electronic subsystem. These couplings determine, for instance, how fast it is possible to change the magnetic state of a material and how the relaxation of phonon and electronic subsystems proceed after excitation with ultrashort laser pulses [13]. In addition, the couplings may shine light on the angular momentum and energy transfer between the spin and lattice subsystems in pump-probe experiments.

A formalism allowing coupled spin-lattice simulations was already provided in the original work on the equations of motion of atomic moments [4]. Recently, such coupling was derived from a different ansatz, where the role of the underlying electronic structure in mediating the coupling is explicitly considered [11]. However, the application to real materials remains a challenge for any formulation of spinlattice simulations.

As already pointed out, dissipation is one of the consequences of the spin-lattice coupling. Since the electron motion is several orders of magnitude faster than both spin and lattice motion, it can, for some purposes, be integrated out $[4,6,8]$. The spin and lattice degrees of freedom, however, occur at a much slower and roughly equivalent time scale and need to be addressed in a unified way, self-consistently $[4,14,15]$. Figure 1 shows a schematic picture of coupled spinlattice dynamics. The exchange interactions between atoms in 


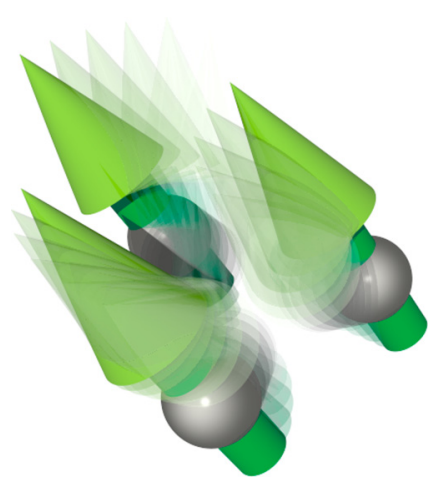

FIG. 1. Conceptual figure of the breathing mode in a trimer of magnetic moments. The spins and atoms are represented as green arrows and gray spheres, respectively.

several magnetic materials can depend strongly on the local atomic environment and vice versa; the chemical interaction may depend on magnetic configuration [16-19]. Hence, both the magnon and phonon spectrum and lifetimes in a material may depend on the configuration of the magnetic state or the displacement of atoms [20,21].

Several studies point at the importance of phononmagnon coupling in a number of dynamical processes such as demagnetization processes [22-24], thermal conductivity [25,26], magnetoacoustics [27-29], and the spin-Seebeck effect [30-32]. The interaction between spin and lattice motion is also central for phenomena observed in magnetoelectric and in multiferroic materials [33-41], magnetocaloric materials [42], skutterudites [43], and antiferromagnetic insulator materials for spintronic devices [44]. Recent developments on methods for modelling of phonon-magnon coupling include (i) a novel combination of atomistic spin dynamics and ab initio molecular dynamics, applied to the paramagnetic phase of the magnetic semiconductor $\mathrm{CrN}$ [45], (ii) a scheme for massively parallel symplectic integration of spin-lattice dynamics equations of motion [46], and (iii) the interplay of nonlinear phononics and exchange striction that opens up for ultrafast control of magnetic ordering through the excitation of the lattice [47].

In the present work, we describe a general method for the simulation of coupled spin-lattice dynamics, where all information needed for the effective spin-lattice dynamics Hamiltonian can be obtained from first-principles theory. We demonstrate the accuracy of the method by applying it to bcc $\mathrm{Fe}$, as well as a selection of smaller clusters. The developed method is based on an atomistic approach and draws its strengths from the atomistic spin dynamics framework. In philosophy, the method proposed here is similar to the early formulation of Ref. [4], but the practical details are naturally different. The interactions are described using a general Hamiltonian, with parameters computed using density functional theory. This hopefully provides a tool for analysis and even prediction of complex collective modes of magnetic materials that is a complement to experimental activities addressing these questions, for instance inelastic neutron scattering (INS) [48] and resonant inelastic x-ray scattering (RIXS) [49-51]. We note that the instrumentation and capabilities of these spectroscopies undergo a rapid development, for instance in form of prismatic analysers for neutron spectrometers [52] for use in the CAMEA instrument [53] at the Paul-Scherrer Institute and in the BIFROST instrument ${ }^{1}$ commissioned for the European Spallation Source (ESS), and furthermore that INS and RIXS are complementary techniques that enable characterization of excitations throughout large parts of the Brillouin zone [54].

Using an empirical potential approach [55,56], spin-lattice dynamics simulations of bcc Fe have been published by several groups [15,57-59]. To put our method in perspective and on firm quantitative ground, we therefore specifically address the spin-lattice interaction in bcc Fe as a test case.

The paper is organized as follows. In Sec. II, we describe the Hamiltonian for coupled spin-lattice dynamics and the associated coupled equations of motion, techniques for calculation of the adiabatic magnon and phonon spectra, and a scheme for numerical integration of the coupled equations of motion. Section III begins with a discussion of the dynamics of magnetic dimers, trimers, and tetramers, and continues with the results for bcc Fe. Finally, in Sec. IV, we discuss the applicability of our method and give an outlook.

\section{MODEL AND METHODS}

This section is split into five parts. First (Sec. II A), we discuss the underlying Hamiltonian, which includes couplings within the spin and lattice reservoirs, respectively, and interactions between the spins and the lattice. Then, the couplings between the spin and lattice degrees of freedom are discussed in more detail in Sec. II B. We motivate the equations-ofmotion and the corresponding observables in Secs. IIC and II D, respectively. In Sec. IIE, we describe how the coupling constants are obtained from density functional theory calculations, and finally we conclude this method section with a brief summary in Sec. II F. We apply the Einstein notation for double occurring Greek indices.

\section{A. The spin-lattice Hamiltonian}

We consider the parametrized Hamiltonian

$$
\mathcal{H}_{\mathrm{SLD}}=\mathcal{H}_{\mathrm{S}}+\mathcal{H}_{\mathrm{L}}+\mathcal{H}_{\mathrm{LS}}
$$

formulated in terms of atomic magnetic moments $\left\{\mathbf{m}_{i}\right\}$, ionic displacements $\left\{\mathbf{u}_{k}\right\}$ and velocities $\left\{\mathbf{v}_{k}\right\}$. The first term is a Hamiltonian describing purely magnetic interactions. The second term contains energies associated with pure lattice displacements, and the third term couples the spin and lattice degrees of freedom. Indices $i$ and $j$ run over atoms $1, \ldots, N_{\mathrm{mag}}$ with a finite magnetic moment, whereas indices $k$ and $l$ run over all the atoms in the simulation cell $1, \ldots, N_{\text {all }}$, i.e., nonmagnetic as well as magnetic ions. Note that in the examples considered, all ions are magnetic. Atomic magnetic moments $\mathbf{m}_{i}$ are in units of Bohr magnetons $\mu_{\mathrm{B}}$, displacements $\mathbf{u}_{k}$ in units of Ångström, and velocities $\mathbf{v}_{k}$ in units of $\AA /$ s. Furthermore, $\alpha, \beta \in\{x, y, z\}$ denote the Cartesian components in spin

\footnotetext{
${ }^{1}$ BIFROST: https://europeanspallationsource.se/article/bifrostprismatic-approach-neutron-spectroscopy.
} 
space, and $\mu, v \in\{x, y, z\}$ denote the Cartesian components in real space.

In the following, we consider contributions up to a combined order of four. The summations over each Latin index $i, j, k, l$ run from 1 to $N$, wherefore factors $1 / 2$ are preceding the coupling constants to account for the double counting. The harmonic approximation to lattice dynamics is described by

$$
\mathcal{H}_{\mathrm{LL}}=\frac{1}{2} \sum_{k l} \Phi_{k l}^{\mu \nu} u_{k}^{\mu} u_{l}^{v}+\frac{1}{2} \sum_{k} M_{k} v_{k}^{\mu} v_{k}^{\mu},
$$

where the force constant tensor $\Phi_{k l}^{\mu \nu}$ is a rank 2 tensor in real space and $M_{k}$ is the mass of atom $k$. Since $\Phi_{k l}^{\mu \nu}$ depends also on the magnetic configuration one can extend the expression above to

$$
\begin{aligned}
\mathcal{H}_{\mathrm{LL}}= & \frac{1}{2} \sum_{k l} \Phi_{k l}^{\mu \nu} u_{k}^{\mu} u_{l}^{\nu}+\frac{1}{2} \sum_{i k l} \frac{\partial \Phi_{k l}^{\mu \nu}}{\partial m_{i}^{\alpha}} m_{i}^{\alpha} u_{k}^{\mu} u_{l}^{v} \\
& +\frac{1}{4} \sum_{i j k l} \frac{\partial^{2} \Phi_{k l}^{\mu \nu}}{\partial m_{i}^{\alpha} \partial m_{j}^{\beta}} m_{i}^{\alpha} m_{j}^{\beta} u_{k}^{\mu} u_{l}^{\nu}+\frac{1}{2} \sum_{k} M_{k} v_{k}^{\mu} v_{k}^{\mu} .
\end{aligned}
$$

For some materials, the force constants depend significantly on the spin configuration as well as the configuration of nuclei. As an example we note that the traditional explanation for Invar alloys relies on the coupling between the force constants and the spin configuration [60], as well as materials where many-body terms of the description of nuclear motion are needed. The main purpose of this paper is to outline a general formalism of coupled spin-lattice dynamics and to give examples of how the coupling modifies the dynamical properties in a few selected cases. In the illustration of the method, we have for simplicity neglected the second and third terms on the right-hand side of Eq. (3), and instead kept a coupling term that originates from the Taylor expansion of exchange parameters (see below). The importance of various contributions to the coupling is materials dependent, but one may note that higher order contributions in the Taylor expansion are expected to be smaller.

The bilinear spin Hamiltonian $\mathcal{H}_{\mathrm{SS}}$ contains Heisenberg exchange, Dzyaloshinskii-Moriya interaction and symmetric, anisotropic interactions that in a compact form can be expressed as

$$
\mathcal{H}_{\mathrm{SS}}=-\frac{1}{2} \sum_{i j} \mathcal{J}_{i j}^{\alpha \beta}\left(\left\{u_{k}^{\mu}\right\}\right) m_{i}^{\alpha} m_{j}^{\beta} .
$$

The exchange tensor $\mathcal{J}_{i j}^{\alpha \beta}$ is a rank 2 tensor in spin space, with elements that in general have a dependence on the atomic displacements $\left\{u_{k}^{\mu}\right\}$ as well as the magnetic configuration. For clarity, we specify in Eq. (4) the explicit dependence of tensor $\mathcal{J}_{i j}^{\alpha \beta}$ on $\left\{u_{k}^{\mu}\right\}$. The contributions to the mixed spinlattice Hamiltonian $\mathcal{H}_{\text {LS }}$ can then be obtained by expanding the bilinear magnetic Hamiltonian $\mathcal{H}_{\mathrm{SS}}$ up to second order in displacement, i.e.,

$$
\begin{aligned}
\mathcal{H}_{\mathrm{SS}}= & -\frac{1}{2} \sum_{i j} \mathcal{J}_{i j}^{\alpha \beta} m_{i}^{\alpha} m_{j}^{\beta}-\frac{1}{2} \sum_{i j k} \frac{\partial \mathcal{J}_{i j}^{\alpha \beta}}{\partial u_{k}^{\mu}} u_{k}^{\mu} m_{i}^{\alpha} m_{j}^{\beta} \\
& -\frac{1}{4} \sum_{i j k l} \frac{\partial^{2} \mathcal{J}_{i j}^{\alpha \beta}}{\partial u_{k}^{\mu} \partial u_{l}^{\nu}} u_{k}^{\mu} u_{l}^{\nu} m_{i}^{\alpha} m_{j}^{\beta} .
\end{aligned}
$$

Note that both in Eqs. (5) and (3) a term enters that contains bilinear couplings in both spin and lattice displacement. In one case, it appears due to Taylor expansion of the Heisenberg exchange parameter in lattice displacement, and in the other, it appears due to a Taylor expansion of the force constant in magnetic moments. In general, both contributions are additive, as they have similar mathematical form. The relative importance of these two contributions is materials dependent and should preferably be calculated for the material one wants to investigate. The four-body interaction accounts for the renormalization of the phonon dispersion due to the spin configuration [36]. It also results in the renormalization of the magnon dispersion due to atomic displacements and enables photon absorption by phonon-assisted multimagnon excitation [61-63].

Introducing the coupling constant $\Gamma_{i j k}^{\alpha \beta \mu}=\partial \mathcal{J}_{i j}^{\alpha \beta} / \partial u_{k}^{\mu}$ we may write the three-body interaction as

$$
\mathcal{H}_{\mathrm{SSL}}=-\frac{1}{2} \sum_{i j k} \Gamma_{i j k}^{\alpha \beta \mu} m_{i}^{\alpha} m_{j}^{\beta} u_{k}^{\mu} .
$$

This represents a spin-lattice coupling which is bilinear in spin and linear in displacement, i.e., $\Gamma_{i j k}^{\alpha \beta \mu}$ is a rank 3 tensor given by the direct product of a rank 2 tensor in spin space and a rank 1 tensor in orbital space. reads

Defining $\Lambda_{i j k l}^{\alpha \beta \mu \nu}=\partial^{2} \mathcal{J}_{i j}^{\alpha \beta} / \partial u_{k}^{\mu} \partial u_{l}^{v}$ the four-body interaction

$$
\mathcal{H}_{\mathrm{SSLL}}=-\frac{1}{4} \sum_{i j k l} \Lambda_{i j k l}^{\alpha \beta \mu v} m_{i}^{\alpha} m_{j}^{\beta} u_{k}^{\mu} u_{l}^{v},
$$

where the factor $1 / 4$ is due to that this interaction is bilinear both in spin and in displacements.

Taken together, the combined spin-lattice Hamiltonian reads

$$
\begin{aligned}
\mathcal{H}_{\mathrm{SLD}}= & -\frac{1}{2} \sum_{i j} \mathcal{J}_{i j}^{\alpha \beta} m_{i}^{\alpha} m_{j}^{\beta}-\frac{1}{2} \sum_{i j k} \Gamma_{i j k}^{\alpha \beta \mu} u_{k}^{\mu} m_{i}^{\alpha} m_{j}^{\beta} \\
& -\frac{1}{4} \sum_{i j k l} \Lambda_{i j k l}^{\alpha \beta \mu \nu} u_{k}^{\mu} u_{l}^{v} m_{i}^{\alpha} m_{j}^{\beta}+\frac{1}{2} \sum_{k l} \Phi_{k l}^{\mu \nu} u_{k}^{\mu} u_{l}^{v} \\
& +\frac{1}{2} \sum_{k} M_{k} v_{k}^{\mu} v_{k}^{\mu} .
\end{aligned}
$$

We note that in order to be even more general, higher coupling such as biquadratic exchange [63-65], four-ring exchange [66], as well as third- and fourth-order [67] interatomic lattice potential can be added, which is relatively straight-forward to do, and that for a very accurate description of the magnetic exchange dependence on the magnetic configuration needs to be considered [68]. Electrostatic contributions to the interatomic force field is also a relevant generalization to consider, since they can be important in polar materials, especially for excitations close to the zone center [69]. Likewise, magnetostatic interactions are sometimes of relevance. We also note here that in many practical simulations the expression in Eq. (8) becomes simplified. In the results section we will provide simulations for, e.g., bcc Fe, and in this case only a simplified version of Eq. (8) is needed. We describe the Hamiltonian for this case in the Results section III B where simulations for bcc Fe are described. 


\section{B. Exchange striction}

The third-order spin-lattice coupling is considered usually in insulating magnets where the spin texture simultaneously breaks time and spatial reversion. This occurs, for instance, when describing ferroelectric polarization and multiferroic phases [40], and it drives the magnetoelectric response in the electromagnetic field driven dynamics in the $\mathrm{GHz}$ and $\mathrm{THz}$ regime [33-35,37,38].

The isotropic (with regard to spin space) part of the $\Gamma_{i j k}^{\alpha \beta \mu}$ tensor is the exchange striction where the Heisenberg coupling between magnetic moments at $i$ and $j$ is modulated by the displacement of ion $k$. The antisymmetric anisotropic (with regard to spin space) part of the tensor represents the Dzyaloshinskii-Moriya interaction. This coupling will not be further discussed, although the demonstrated implementation in principle allows for it.

In this paper, we focus on the nonrelativistic correction term to Heisenberg exchange

$$
\mathcal{H}_{\mathrm{SSL}}=-\frac{1}{2} \sum_{i j k} \boldsymbol{A}_{i j k} \cdot \mathbf{u}_{k}\left(\mathbf{m}_{i} \cdot \mathbf{m}_{j}\right),
$$

in Eq. (6) and for the simulations presented in Sec. III, where atom $k$ could either coincide with one of the atoms $i$ and $j$ or be a distinct atom. The components of the exchange striction vector $\boldsymbol{A}_{i j k}$ relate to $\Gamma_{i j k}^{\alpha \beta \mu}$ according to $A_{i j k}^{\mu}=\Gamma_{i j k}^{x x \mu}=\Gamma_{i j k}^{y y \mu}=$ $\Gamma_{i j k}^{z z \mu}$.

The exchange striction coupling parameters are governed by certain symmetry rules. From the symmetry of Heisenberg exchange interaction,

$$
\boldsymbol{A}_{i j k}=\boldsymbol{A}_{j i k} .
$$

Furthermore, the sum rule

$$
\boldsymbol{A}_{i j l}=-\sum_{k \neq l} \boldsymbol{A}_{i j k}
$$

must necessarily hold in order to conserve the position of the center of mass. This sum rule is derived by enforcing that the forces emerging from exchange striction sum up to zero, i.e.,

$$
\boldsymbol{F}_{l}=-\sum_{k \neq l} \boldsymbol{F}_{k}
$$

and then using the definition of the exchange striction force

$$
\boldsymbol{F}_{l}=\sum_{i j} \boldsymbol{A}_{i j l}\left(\mathbf{m}_{i} \cdot \mathbf{m}_{j}\right) .
$$

In different model approximations for the Heisenberg exchange, for instance, the Ruderman-Kittel-Kasuya-Yosida (RKKY) type interaction [70] or the effective model used by Ma et al. [71], the exchange interaction depends only on the distance $r_{i j}$ between site $i$ and $j, J_{i j}\left(r_{i j}\right)=J_{i j}\left(\mid \mathbf{R}_{j}+\mathbf{u}_{j}-\right.$ $\mathbf{R}_{i}-\mathbf{u}_{i} \mid$ ), and, consequently, $\boldsymbol{A}_{i j i}=\left|\boldsymbol{A}_{i j i}\right| \mathbf{r}_{i j}$, where $\boldsymbol{r}_{i j}$ is the vector connecting site $i$ and $j$ of the length $r_{i j}$. Only in this case, it reflects the symmetry rule $\boldsymbol{A}_{i j i}=-\boldsymbol{A}_{i j j}$. In general, the exchange interaction does not need to depend only on the distance $r_{i j}$ between the two sites $i$ and $j$. Consequently, this symmetry relation must not necessarily be true in general. However, the relation $\boldsymbol{A}_{i j i}=-\boldsymbol{A}_{i j j}$ guarantees that the exchange striction force on atom $i$ and $j$ will cancel each other.
The direction of the force caused by the exchange striction coupling is contained in $\boldsymbol{A}_{i j k}$ and the directions of different $\boldsymbol{A}_{i j k}$ are related by the point group symmetry of the crystal.

\section{The SLD equations of motion}

The coupled equations of motion for the spin-lattice system reads [15]

$$
\begin{aligned}
& \frac{d \mathbf{m}_{i}}{d t}=-\frac{\gamma}{\left(1+\alpha^{2}\right)} \mathbf{m}_{i} \times\left(\mathbf{B}_{i}+\mathbf{B}_{i}^{\mathrm{fl}}\right) \\
&- \frac{\gamma}{\left(1+\alpha^{2}\right)} \frac{\alpha}{m_{i}} \mathbf{m}_{i} \times\left(\mathbf{m}_{i} \times\left[\mathbf{B}_{i}+\mathbf{B}_{i}^{\mathrm{fl}}\right]\right), \\
& \frac{d \mathbf{u}_{k}}{d t}=\mathbf{v}_{k}, \\
& \frac{d \mathbf{v}_{k}}{d t}=\frac{\mathbf{F}_{k}}{M_{k}}+\frac{\mathbf{F}_{k}^{\mathrm{fl}}}{M_{k}}-v \mathbf{v}_{k},
\end{aligned}
$$

when expressed in the form of Langevin equations. Here, the effective magnetic field is obtained from the SLD Hamiltonian in Eq. (1) as $\mathbf{B}_{i}=-\partial \mathcal{H}_{\mathrm{SLD}} / \partial \mathbf{m}_{i}$ and the effective interatomic force field is determined from $\mathbf{F}_{k}=-\partial \mathcal{H}_{\mathrm{SLD}} / \partial \mathbf{u}_{k}$. $M_{k}$ is the mass of the atom at site $k, m$ is the saturation magnetization, $\gamma$ is the gyromagnetic ratio, while $\alpha$ and $v$ are scalar (isotropic) damping constants. The stochastic fields $\mathbf{B}_{i}^{\mathrm{fl}}$ and $\mathbf{F}_{k}^{\mathrm{fl}}$ are modelled as white noise with properties $\left\langle B_{i, \mu}^{\mathrm{fl}}(t) B_{j, \nu}^{\mathrm{fl}}\left(t^{\prime}\right)\right\rangle=2 D_{M} \delta_{i j} \delta_{\mu \nu} \delta\left(t-t^{\prime}\right)$ and $\left\langle F_{i, \mu}^{\mathrm{fl}}(t) F_{j, \nu}^{\mathrm{fl}}\left(t^{\prime}\right)\right\rangle=$ $2 D_{L} \delta_{k l} \delta_{\mu \nu} \delta\left(t-t^{\prime}\right)$. From the fluctuation-dissipation theorem it follows that $D_{M}=\alpha k_{B} T / \gamma m$ and $D_{L}=v M k_{B} T$ [15], where $T$ is the temperature and $k_{B}$ is the Boltzmann constant.

The coupled partial differential equations are numerically solved using the semi-implicit SIB method by Mentink et al. [72] combined with the Grønbech-Jensen-Farago Verlet-type method [73], or with a fixed-point scheme for implicit midpoint method. The methods require numerical step width of the order $10^{-15}$ down to $10^{-16} \mathrm{~s}$. Details about the algorithms are provided in Appendix A.

\section{Observables}

The primary output of a spin-lattice dynamics simulation are trajectories in time of the system variables $\left\{\mathbf{m}_{i}\right\},\left\{\mathbf{u}_{k}\right\}$, and $\left\{\mathbf{v}_{k}\right\}$. In order to sample spatial and temporal fluctuations of the spins and the ions, we define the space- and time-displaced pair correlation functions

$$
\begin{aligned}
C_{(\mathbf{m})}^{\alpha \beta}\left(\mathbf{r}, t-t_{0}\right) & =\frac{1}{N_{\mathrm{mag}}} \sum_{\substack{i, j \text { where } \\
\mathbf{r}_{i}-\mathbf{r}_{j}=\mathbf{r}}}\left\langle m_{i}^{\alpha}(t) m_{j}^{\beta}\left(t_{0}\right)\right\rangle, \\
C_{(\mathbf{u})}^{\mu v}\left(\mathbf{r}, t-t_{0}\right) & =\frac{1}{N_{\mathrm{all}}} \sum_{\substack{k, l \text { where } \\
\mathbf{r}_{k}-\mathbf{r}_{l}=\mathbf{r}}}\left\langle u_{k}^{\mu}(t) u_{l}^{v}\left(t_{0}\right)\right\rangle, \\
C_{(\mathbf{v})}^{\mu v}\left(\mathbf{r}, t-t_{0}\right) & =\frac{1}{N_{\mathrm{all}}} \sum_{\substack{k, l \text { where } \\
\mathbf{r}_{k}-\mathbf{r}_{l}=\mathbf{r}}}\left\langle v_{k}^{\mu}(t) v_{l}^{v}\left(t_{0}\right)\right\rangle .
\end{aligned}
$$

Equations (17)-(19) can thus describe how the magnetic, displacement, and velocity correlations evolve both in space and over time. It would of course be natural to investigate coupled 
modes, via coupled correlations, e.g., between moment and displacement in a similar way as outlined in Eqs. (17)-(19). In the context of the simulations of bcc Fe in the present paper, the more relevant property is however obtained by a Fourier transform over space and time to give the dynamic structure factor for spin, displacement, and velocities. Defining relative time $\tau=t-t_{0}$, we obtain

$$
S_{(\mathbf{m})}^{\alpha \beta}(\mathbf{Q}, \omega)=\frac{1}{N_{\mathrm{mag}} \sqrt{2 \pi}} \sum_{\mathbf{r}} e^{i \mathbf{Q} \cdot \mathbf{r}} \int_{-\infty}^{\infty} e^{i \omega \tau} C_{(\mathbf{m})}^{\alpha \beta}(\mathbf{r}, \tau) d \tau,
$$

$$
S_{(\mathbf{u})}^{\mu \nu}(\mathbf{Q}, \omega)=\frac{1}{N_{\mathrm{all}} \sqrt{2 \pi}} \sum_{\mathbf{r}} e^{i \mathbf{Q} \cdot \mathbf{r}} \int_{-\infty}^{\infty} e^{i \omega \tau} C_{(\mathbf{u})}^{\mu \nu}(\mathbf{r}, \tau) d \tau,
$$

$$
S_{(\mathbf{v})}^{\mu v}(\mathbf{Q}, \omega)=\frac{1}{N_{\mathrm{all}} \sqrt{2 \pi}} \sum_{\mathbf{r}} e^{i \mathbf{Q} \cdot \mathbf{r}} \int_{-\infty}^{\infty} e^{i \omega \tau} C_{(\mathbf{v})}^{\mu v}(\mathbf{r}, \tau) d \tau,
$$

which are closely related to what is measured by inelastic neutron or electron scattering experiments. The dynamic structure factors are naturally analyzed in terms of the differential cross section [48], which for many materials is proportional to the dynamical structure function. This means that by simulating the dynamical structure factor, the relation between momentum transfer $\mathbf{Q}$ and frequency $\omega$ for magnons and phonons in the material can be obtained.

For systems lacking periodicity, such as the small dimer, trimer, and tetramer clusters also considered in this work, the $\mathbf{Q}$ dependence of the structure factor is in principle undefined. Instead, the spatially dependent excitation spectra of these finite systems can be obtained by performing the Fourier transform over time only. Summing the resulting correlation function over all sites results in the total excitation spectra of the system.

For the finite clusters, we only consider the components of the magnetic correlation functions that are perpendicular to the starting state of the magnetization. This is done to increase numerical stability.

The spin dynamical structure factor accurately describe magnon dispersions, especially in thin films [74], since it properly takes into account magnon-magnon scattering properties and damping at finite temperatures. On the other hand, in the limit of very low temperatures and damping, the magnon dispersion is more easily obtained through adiabatic linear spin wave theory [75].

Let us first focus on the spin degrees of freedom and the adiabatic magnon spectra for the collinear magnetic case with a system consisting of 1 atom/cell such as bcc Fe. Then the spatial Fourier transform of the exchange interactions reads

$$
J(\mathbf{Q})=\sum_{j \neq 0} J_{0 j} e^{i \mathbf{Q} \cdot\left(\mathbf{R}_{j}-\mathbf{R}_{0}\right)},
$$

where $J_{0 j}$ is the isotropic exchange interaction between magnetic atoms at site 0 and $j$, respectively. Note that here the index $j$ runs over all magnetic sites with the origin at $\mathbf{R}_{0}$. The spin wave energies $\omega(\mathbf{Q})$ will then be given by the following expression [76-78]:

$$
\omega(\mathbf{Q})=\gamma m(J(\mathbf{0})-J(\mathbf{Q})) .
$$

Generalization can be done towards multisublattice systems, see, e.g., Ref. [8], and using Bogoliubov transformation [79] towards general noncollinear formulation $[80,81]$.

For the lattice degrees of freedom, the reciprocal space dynamical matrix $D_{s t}^{\mu \nu}(\mathbf{Q})$ is related to the force constant matrix in real space by the mass normalised Fourier transform

$$
D_{s t}^{\mu \nu}(\mathbf{Q})=\frac{1}{\sqrt{M_{s} M_{t}}} \sum_{l} \Phi_{0 s, l t}^{\mu v} e^{\left[i \mathbf{Q} \cdot\left(\mathbf{R}_{l}-\mathbf{R}_{0}\right)\right]}
$$

where $M_{s}$ is the mass of atom $s$ in the unit cell. Given the translational symmetry of the crystal, it is enough to sum only over $l$ in all the $N_{P}$ primitive cells in the supercell. For the $\Gamma$ point, the Fourier transform is a plain sum over all repetitions of the primitive cell, both the ones contained in the WignerSeitz cell of the simulation supercell and, due to the periodic boundary conditions, the ones outside it $[82,83]$. Solving the eigenvalue problem [83]

$$
\operatorname{det}\left[\mathbf{D}(\mathbf{Q})-\omega^{2}(\mathbf{Q}) \mathbf{l}\right]=0,
$$

the $3 N$ phonon modes (eigenvectors) and frequencies (the square roots of the occasionally degenerate eigenvalues $\omega^{2}$ ) are obtained for a given $\mathbf{Q}$ vector. Here, I is the unit matrix. In contrast to the dynamic structure factor [Eqs. (20)-(22)], the adiabatic spectra described above do not directly account for the coupling between the spin and lattice reservoir. A possible way forward in this regard is to replace the exchange interaction and force constant matrix in Eqs. (23) and (25), with corresponding Taylor expanded entities defined in Eqs. (5) and (3), respectively. Analytical methods for investigations of the excitation spectra of spin-lattice coupled Hamiltonians have been used, e.g., in Refs. [54,84-86], but they are beyond the scope of the present paper.

\section{E. DFT calculations}

Aiming for a first-principles description of the coupled spin-lattice dynamics in bcc Fe, we calculated the coupling constants that occur in Eq. (8) through density functional theory (DFT) calculations.

The harmonic force constants $\Phi_{k l}^{\mu \nu}$ were calculated with the finite displacement method using the Vienna ab initio Vienna simulation package (VASP) $[87,88]$ and the PHONOPY [89] software. The VASP calculations were performed using the projector augmented wave method $[90,91]$ and the local density approximation as exchange-correlation functional. A $6 \times 6 \times 6$ supercell was used for bcc Fe. We employed a plane-wave energy cutoff of $600 \mathrm{eV}$ and a $\Gamma$-centered $k$-point mesh of size $4 \times 4 \times 4$.

In order to achieve a complete first-principles spin-lattice model, we also extract the Heisenberg interaction and the exchange striction from DFT. To this end, we applied the full-potential linear muffin-tin orbitals (FP-LMTO) method as implemented in the RSPt software [92]. The maximum value of the angular momentum used for the angular $(l)$ decomposition of the charge density and the potential inside the muffin-tin spheres was taken equal to $l_{\max }=12$. Three kinetic energy tails were used for the description of the states in the interstitial region: $-0.3,-2.3$, and $-1.5 \mathrm{Ry}$. 
Within this setup, we calculated the Heisenberg exchange coupling $J_{i j}$ via the Liechtenstein-Katsnelson-Antropov-Gubanov (LKAG) formalism [93,94]. For the actual implementation into the RSPT code, we refer the reader to Ref. [95]. Recently, this method has been successfully applied to strongly correlated systems such as $\mathrm{NiO}$ [95] and $\mathrm{BiFeO}_{3}$ [96].

The magnetic exchange interactions $J_{i j}$ have been calculated with RSPt for a primitive cell and for a $2 \times 2 \times 2$ supercell for which identical $J_{i j}$ were obtained. In order to calculate first-order exchange striction, $J_{i j}$ have been calculated for the same $2 \times 2 \times 2$ cell but with one atom displaced with a finite displacement $\Delta$ along $\boldsymbol{e}_{\Delta}$. A $k$-point mesh of $30 \times 30 \times 30$ was employed for the supercell calculations. The so obtained set of exchange couplings have a lower symmetry than the exchange couplings obtained for the undistorted supercell. For instance, the set of eight equivalent nearest-neighbor couplings in the bcc structure are broken up to sets of 1, 3, 3,1 degenerate couplings for a distortion along the [100] axis (in bcc lattice vectors, i.e., along the [111] for Cartesian axis), and sets of 4, 4 couplings for a distortion along the [110] axis (Cartesian [100]). For the bcc structure, we have compared carefully that for different distortion directions, the symmetry lowering for the $J_{i j}$ up to the fourth coordination shell is identical to the symmetry lowering of the crystal itself.

Since anisotropic exchange parts are not considered, the tensor $\boldsymbol{\Gamma}_{i j k}$ reduces to a vector $\boldsymbol{A}_{i j k}$. Similar to the force constants and magnetic exchange, $\boldsymbol{A}_{i j k}$ fulfill point group symmetries, in particular for bcc Fe the 48 symmetry operations of space group number $229(\operatorname{Im} \overline{3} m)$. Furthermore, the exchange striction energy $E_{i j k}$ related to the sites $i, j$, and $k$ is isotropic. Consequently, we obtain $\boldsymbol{A}_{i j k}=\boldsymbol{A}_{j i k}$ from $E_{i j k}=E_{j i k}$, which is caused by the isotropic properties of the magnetic exchange. In our spin-lattice dynamics simulations, we enforce that the center of mass is not drifting, which can be achieved by applying the appropriate sum rule, see Eq. (11).

Using a finite difference method involving the nondisplaced set $\left\{J^{0}\right\}$ and a displaced set $\left\{J^{e_{\Delta}}\right\}$, we obtain the directional derivative $\Gamma_{i j k}^{\Delta}$. The gradient $\nabla_{k} J_{i j}$ is finally constructed from the directional derivative definition and out of three different sets $\left\{J_{\Delta}^{v}\right\}$ of independent directions $\boldsymbol{e}_{\Delta}^{\nu}$, where $v=$ $1,2,3$, but the same displacement strength $\Delta$. We have chosen $\Delta$ to be $0.003 a_{0}, 0.002 a_{0}, 0.001 a_{0}$, and $0.00001 a_{0}$, where $a_{0}$ is the Bohr radius. In order to fulfill the finite displacement criteria, $\Delta$ is interpolated to zero by Hermite interpolation. Numerical noise in $\Gamma_{i j k}^{\Delta}$ is reduced by enforcing the fulfillment of the correct group symmetries, as discussed above. We iteratively apply the above mentioned symmetry operations until we reach convergence. In the last step, conservation of the position of the center of mass is enforced through the use of Eq. (11).

\section{F. Summary of the methods}

Summarizing Sec. II, we present a parametrized Hamiltonian that describes a material in which the spin and lattice dynamics are coupled. We also provide the necessary equations of motion that are to be solved for coupled spin-lattice dynamics simulations and we outline how coupling terms may be evaluated from first principles theory. We also provide a description on how to extract observables from such simulations, which one may compare with experimentally measured data.
We emphasize the difference between the method presented here and the one proposed by Ma et al. $[15,57]$ and Perera et al. [59]. (i) All sets of parameters $\{J\},\{\Phi\}$, and $\{\Gamma\}$ are determined from first principles and are not obtained from the effective potential or exchange model. (ii) We consider an established parametrization of the lattice potential as presented in Eq. (2), which is directly available from standard first-principles tools (see Sec. II E). (iii) the exchange striction term contains also couplings for $k \neq i, j$ and, consequently, will be highly applicable for magnets showing super- and double exchange mechanism for the magnetic coupling.

\section{RESULTS}

This section is divided into two parts. In Sec. III A, we discuss the application of our method to low-dimensional model systems and discuss symmetry-related issues of the three-body exchange coupling. (Note that we have selected, for simplicity, to neglect four-body interactions in the present work.) Section III B deals with applications to real materials, in particular to bcc Fe. All required parameters for the bcc Fe Hamiltonian are calculated from first principles. We present quasiparticle dispersion relations and discuss the role of the three-body interactions in the spectra at various temperatures.

\section{A. Exchange striction in two-, three-, and four-site systems}

As conceptual examples for our method, we perform coupled spin-lattice dynamics simulations for systems consisting of two (dimer), three (trimer), and four (tetramer) atoms. Unless explicitly mentioned, we neglect energy dissipation in our model and, consequently, the total energy has to be conserved. Furthermore, we account only for the isotropic part of the magnetic exchange tensor, namely, the Heisenberg exchange, but note that anisotropy in general is of significant importance in low-dimensional systems [74,97-101].

\section{Dimer}

In this model system, we consider a dimer where the two sites are denoted by 1 and 2 (see the inset of Fig. 2) and we have set $M_{1}=M_{2}=10$ atomic mass units and $m_{1}=m_{2}=$ $1 \mu_{B}$. The simplicity of this system allows to provide explicit expressions for the Hamiltonian, the effective magnetic fields and the interatomic forces. The four parts of the dimer Hamiltonian

$$
\mathcal{H}^{\text {dimer }}=\mathcal{H}_{\mathrm{LL}}^{\text {dimer }}+\mathcal{H}_{\mathrm{SS}}^{\text {dimer }}+\mathcal{H}_{\mathrm{SSL}}^{\text {dimer }}+\mathcal{H}_{\mathrm{KIN}}^{\text {dimer }}
$$

read

$$
\begin{aligned}
& \mathcal{H}_{\mathrm{LL}}^{\text {dimer }}= \frac{1}{2} \Phi_{11}^{\mu \nu} u_{1}^{\mu} u_{1}^{\nu}+\frac{1}{2} \Phi_{12}^{\mu \nu} u_{1}^{\mu} u_{2}^{\nu}+\frac{1}{2} \Phi_{21}^{\mu \nu} u_{2}^{\mu} u_{1}^{\nu} \\
&+\frac{1}{2} \Phi_{22}^{\mu \nu} u_{2}^{\mu} u_{2}^{\nu}, \\
& \mathcal{H}_{\mathrm{SS}}^{\text {dimer }}=-\frac{1}{2} J_{12} \mathbf{m}_{1} \cdot \mathbf{m}_{2}-\frac{1}{2} J_{21} \mathbf{m}_{2} \cdot \mathbf{m}_{1}, \\
& \mathcal{H}_{\mathrm{SSL}}^{\text {dimer }}=-\frac{1}{2} A_{121}^{\mu}\left(\mathbf{m}_{1} \cdot \mathbf{m}_{2}\right) u_{1}^{\mu}+\frac{1}{2} A_{122}^{\mu}\left(\mathbf{m}_{1} \cdot \mathbf{m}_{2}\right) u_{2}^{\mu} \\
&+\frac{1}{2} A_{211}^{\mu}\left(\mathbf{m}_{2} \cdot \mathbf{m}_{1}\right) u_{1}^{\mu}+\frac{1}{2} A_{212}^{\mu}\left(\mathbf{m}_{2} \cdot \mathbf{m}_{1}\right) u_{2}^{\mu}, \\
& \mathcal{H}_{\mathrm{KIN}}^{\text {dimer }}=\frac{1}{2} M_{1} v_{1}^{\mu} v_{1}^{\mu}+\frac{1}{2} M_{2} v_{2}^{\mu} v_{2}^{\mu} .
\end{aligned}
$$




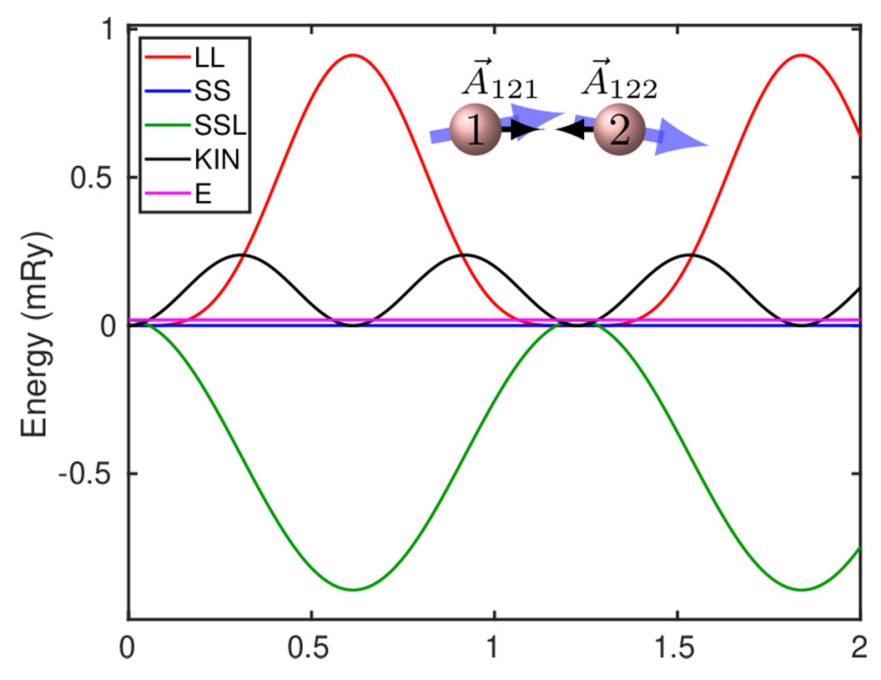

FIG. 2. Energy trajectories at $T=0 \mathrm{~K}$ of a dimer oriented along the $x$ direction with Hamiltonian according to Eqs. (27)-(31). The inset shows a conceptual figure of the exchange striction coupling constants $\boldsymbol{A}_{12 k}, k=1,2$ (black arrows) in a dimer. The atoms are represented by red spheres, where the initial magnetic moment configuration in the dimer is given by blue arrows.

In particular for the dimer, we choose the magnetic interaction to be $J_{12}=1 \mathrm{mRy} \mu_{\mathrm{B}}^{-2}$ and the harmonic atomic force constants uniaxial with $\Phi_{11}^{x x}=-\Phi_{12}^{x x}=-\Phi_{21}^{x x}=\Phi_{22}^{x x}=$ $1 \mathrm{mRy} \AA^{-2}$. The three-body interaction is introduced along the bond and is set to $1 \mathrm{mRy} \mu_{\mathrm{B}}^{-2} \AA^{-1}$. The scalar product of the two moments in the dimer is, when damping is ignored, a constant of motion and hence also the exchange energy will be constant (see Fig. 2 blue line). In the absence of exchange striction we let the atom have ideal molecular positions $\mathbf{r}_{1}=$ $(0,0,0)$ and $\mathbf{r}_{2}=(a, 0,0)$, where the constant $a$ in an actual molecular system can take a value of a few Ångström. Due to the exchange striction, the atoms are shifted from the ideal positions to new equilibrium positions $\mathbf{s}_{1}=(-\Delta u, 0,0)$ and $\mathbf{s}_{2}=(a+\Delta u, 0,0)$ around which they oscillate harmonically. By completing the square for the displacements $\mathbf{u}_{1}$ and $\mathbf{u}_{2}$ in Eq. (27), we obtain $\Delta u=A_{121} \mathbf{m}_{1} \cdot \mathbf{m}_{2} /\left(2 \Phi_{11}\right)=0.47 \AA$ for a starting magnetic configuration with $\mathbf{m}_{1} \cdot \mathbf{m}_{2}=0.94$. The value of $\Delta u$ is here large, partially due to our choice of $A_{121}$ and partially due to that the dimer is an open system.

The evolution of the lattice degrees of freedom is dictated by the corresponding harmonic interatomic forces

$$
F_{\mathrm{LL}, 1}^{\text {dimer }, \mu}=-\Phi_{11}^{\mu \nu} u_{1}^{v}-\Phi_{12}^{\mu \nu} u_{2}^{v},
$$

and interatomic forces from the three-body exchange

$$
F_{\mathrm{SLL}, 1}^{\mathrm{dimer}, \mu}=A_{121}^{\mu}\left(\mathbf{m}_{1} \cdot \mathbf{m}_{2}\right),
$$

where we used the symmetry relations of the force constants $\Phi_{k l}^{\mu \nu}=\Phi_{l k}^{\nu \mu}$ and of the exchange striction terms $A_{121}^{\mu}=A_{211}^{\mu}$.

Likewise, the magnetic degrees of freedom are driven by the magnetic exchange field

$$
\mathbf{B}_{\mathrm{SS}, 1}=J_{12} \mathbf{m}_{2}
$$

and exchange striction field

$$
\mathbf{B}_{\mathrm{SSL}, 1}=A_{121}^{\mu} \mathbf{m}_{2} u_{1}^{\mu}+A_{122}^{\mu} \mathbf{m}_{2} u_{2}^{\mu} .
$$

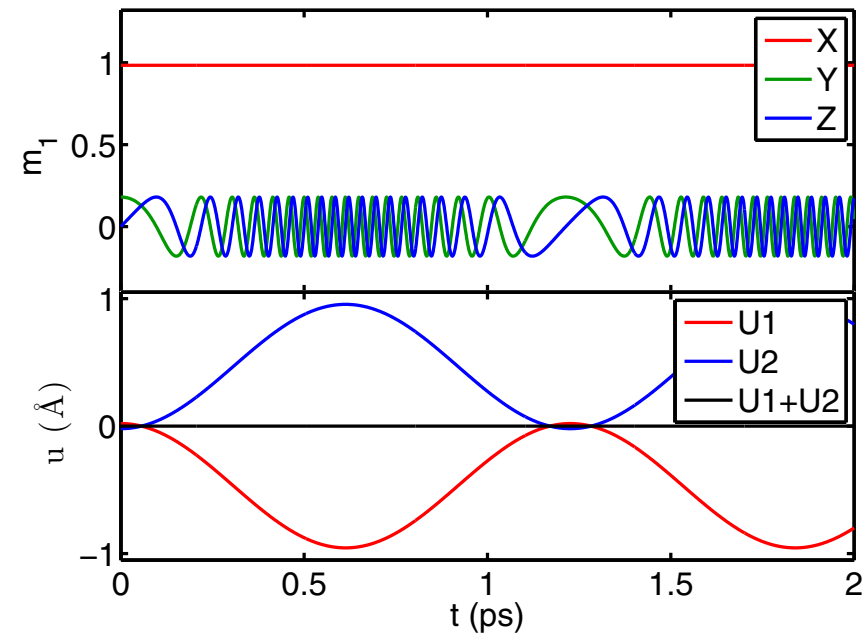

FIG. 3. Trajectories at $T=0 \mathrm{~K}$ of a dimer oriented along the $x$ direction. The slow ionic motion couples over the exchange striction to the spin system and induces a modulation of the frequencies of spin precession. (Top) The Cartesian components of $\mathbf{m}_{1}$, labeled in the figure with $X, Y$, and $Z$. (Bottom) The displacement of ion 1 and 2 along the dimer bond axis.

Here, we applied the isotropy property of the magnetic exchange $J_{12}=J_{21}$. Equations (32)-(35) show that the direction of $\boldsymbol{F}_{\text {SLL, } 1}^{\text {dimer }}$ is only dictated by the coupling constant, where the amplitude is also related to the relative angle between the magnetic moments. Hence, in the absence of Gilbert damping that strives to align the spins, $\boldsymbol{F}_{\text {SLL, } 1}^{\text {dimer }}$ will be also a constant of motion in the case of a dimer. For the effective magnetic field, the three-body term only scales the field strength. The exchange striction term conserves the center of mass and, consequently, $\boldsymbol{u}_{1}=-\boldsymbol{u}_{2}$ and $\boldsymbol{A}_{121}=-\boldsymbol{A}_{122}$. Thus the case $J_{12}<$ $2 \boldsymbol{A}_{121} \cdot \boldsymbol{u}_{1}$ is of high interest, since the effective exchange switches from a ferromagnetically to an antiferromagnetically coupled system. For the dimer, however, this will only change the direction of precession locally in time.

Thus the fields and forces in Eqs. (32)-(35) lead to the time evolution of the spin and lattice degrees of freedom. The different contributions to the energy are shown in Fig. 2, while the time evolution of the displacement and the projections of the magnetic moment are shown in Fig. 3. The simulations reproduce the conservation of the center of mass (Fig. 3 lower panel), as well as the relative angle between the magnetic moments. The initial conditions for the displacement in the dimer are set to be $0.02 \AA$ ( $2 \%$ of the lattice constant $a=1 \AA$ ). It should be noted that the oscillation is not around the molecular positions that are obtained with no exchange striction, $\mathbf{r}_{1}$ and $\mathbf{r}_{2}$, but around the equilibrium positions obtained when exchange striction is considered, i.e. $\mathbf{s}_{1}$ and $\mathbf{s}_{2}$. It should also be noted that the magnetic moments move only in the $y z$ plane as a result of the initial conditions (see Fig. 2, inset, purple arrows). The precession frequency of the magnetic moment is seen to vary in time and to be largest when the displacement $\boldsymbol{u}_{1}$ is significant. This is a natural consequence of the exchange striction field, defined in Eq. (35). A movie that shows the dynamics of spin and nuclear motion of the dimer is shown in Ref. [102]. 


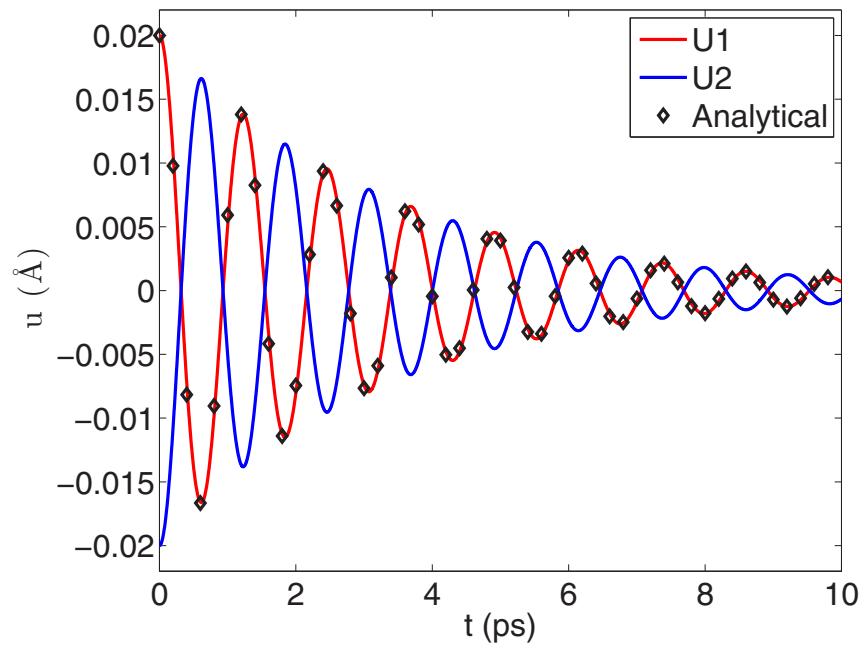

FIG. 4. Trajectories at $T=0 \mathrm{~K}$ of the damped lattice motion of a dimer oriented along the $x$ direction. The analytical solution for atom 1 (black symbols) lies on top of the simulated trajectory (red line).

To further analyze the reliability of our method we also simulate the dynamics in the presence of a viscous damping $v=10^{-14} \mathrm{~s}^{-1}$, and for a weaker exchange striction of $1 \mu \operatorname{Ry} \mu_{B}^{-2} \AA^{-1}$. The analytical solution of the damped 1Dharmonic oscillator (see, e.g., Ref. [103]) is compared with the numerical one and we obtain perfect agreement, as shown in Fig. 4. The ions oscillate around the center of mass and the envelope of the trajectories decays exponentially in time.

\section{Trimer}

The three sites of a trimer are mutually nearest neighbors, which enables for a total of 18 possible $\boldsymbol{A}_{i j k}$ couplings. Respecting the symmetry constraints of the exchange coupling parameters [Eq. (10)] dictated by the point group symmetry $D_{3 h}$ and the fact that the sum rule [Eq. (11)] should always hold, we consider the following case for the exchange striction, as illustrated in Fig. 5. Exchange striction vectors tilt away from the bond by angle $\theta_{1}: \boldsymbol{A}_{i j i} \nmid \mathbf{r}_{i j}$ and, consequently, $\boldsymbol{A}_{i j k(k \neq i, j)} \neq \mathbf{0}$. Furthermore, $\boldsymbol{A}_{i j 1}=-\sum_{l \neq 1} \boldsymbol{A}_{i j l}=$ $-\sum_{l \neq 1} \boldsymbol{A}_{j i l}=\boldsymbol{A}_{j i 1}$. Please note that the equality $\left|\boldsymbol{A}_{i j i}\right|=$

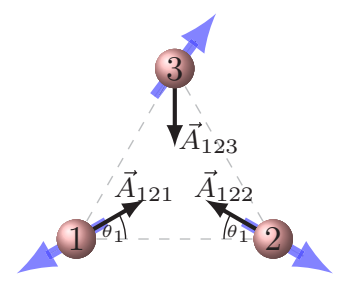

FIG. 5. Conceptual figure of the exchange striction coupling constants $\boldsymbol{A}_{12 k}, k=1,2,3$ (black arrows), in a trimer. By the sum rule Eq. (11), we have $\boldsymbol{A}_{123}=-\boldsymbol{A}_{121}-\boldsymbol{A}_{122}$ and one free angle $\theta_{1}$ as a parameter. The atoms are represented by red spheres, where the initial magnetic moment configuration in the trimer is given by blue arrows. The initial condition of the displacement are chosen arbitrarily.

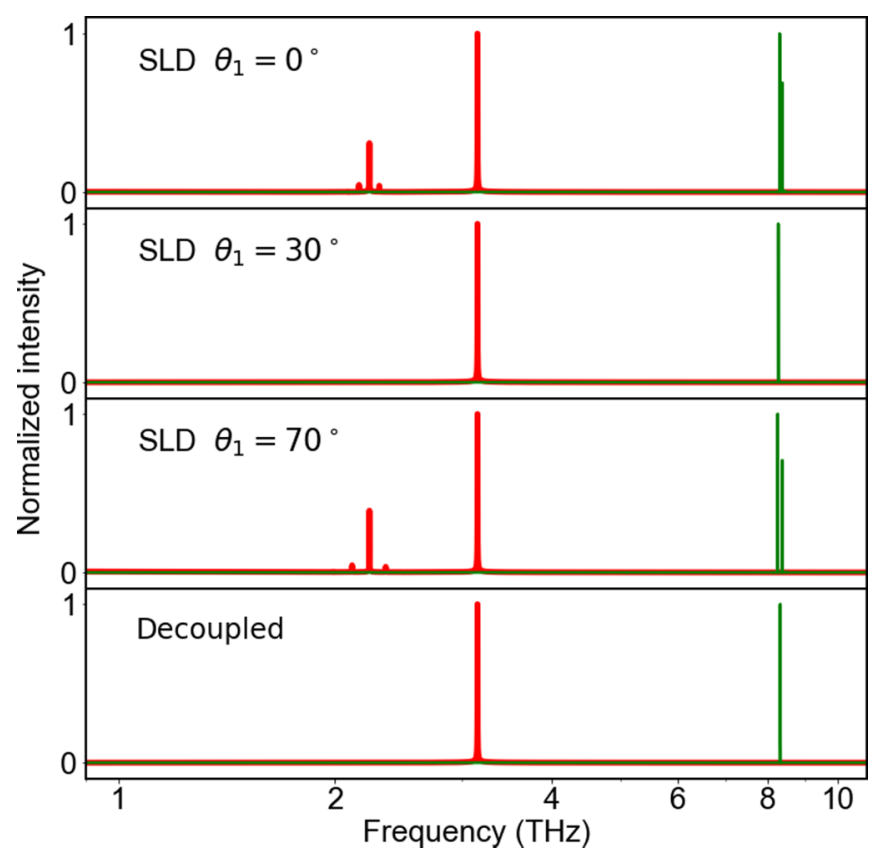

FIG. 6. Excitation spectra for lattice displacements (red/thick lines) and magnetic oscillations (green/thin lines), for the trimer configurations. The top three panels corresponds to SLD simulations with $\theta_{1}=0^{\circ}, 30^{\circ}$, and $70^{\circ}$, as described in the text. The bottom panel corresponds to decoupled lattice-dynamics and spin-dynamics simulations.

$\left|\boldsymbol{A}_{i j k(k \neq i, j)}\right|$ is only fulfilled for $\theta_{1}=\pi / 6$. Also, we here refer to the amplitude of $\boldsymbol{A}_{i j i}$ as $A$.

Just as for the dimer case, we assume nearest-neighbor coupling of $J=1 \mathrm{mRy} \mu_{\mathrm{B}}^{-2}$. The irreducible part of the atomic force constants $\Phi$ for $D_{3 h}$ symmetry are in the notation of Refs. [54,104], i.e., $\Phi_{12}^{x x}=-0.25 \mathrm{mRy} \AA^{-2}, \Phi_{12}^{x y}=$ $-0.43 \mathrm{mRy} \AA^{-2}$, and $\Phi_{12}^{y y}=-0.25 \mathrm{mRy} \AA^{-2}$. The mass of each atom is put to 1 atomic mass unit.

A movie in Ref. [105] shows the dynamics of spinand nuclear motion of the trimer. For clarity we have in the illustration (but not in the actual simulation) exaggerated the amplitude of the nuclear vibrations. Note that due to the exchange striction term, the effective field that governs the spin-dynamics, is coupled to the nuclear motion. This is the main cause why the magnetic moment changes rather abruptly at certain times.

In order to see direct effects of the $A$ coupling, we plot in Fig. 6, the measured excitation spectra given by Eqs. (20) and (21) with $\mathbf{Q}=\mathbf{0}$, obtained from simulations for three choices of $\theta_{1}$ (defined in Fig. 5) with $0.1 \mathrm{mRy} \mu_{\mathrm{B}}^{-2} \AA^{-1}$ For the magnetic correlation function only the parts perpendicular to the starting state of the magnetization are considered, whereas for the displacement correlation function all the Cartesian components are used. Note that $\mathbf{Q}=\mathbf{0}$ is the only relevant choice since there is no translation symmetry of the cluster. For comparison, the excitation spectra for a reference system with $A=0$, i.e., without any coupling between the spin and lattice systems is also included in the figure. For the decoupled trimer, two significant peaks are present. The peak that is 

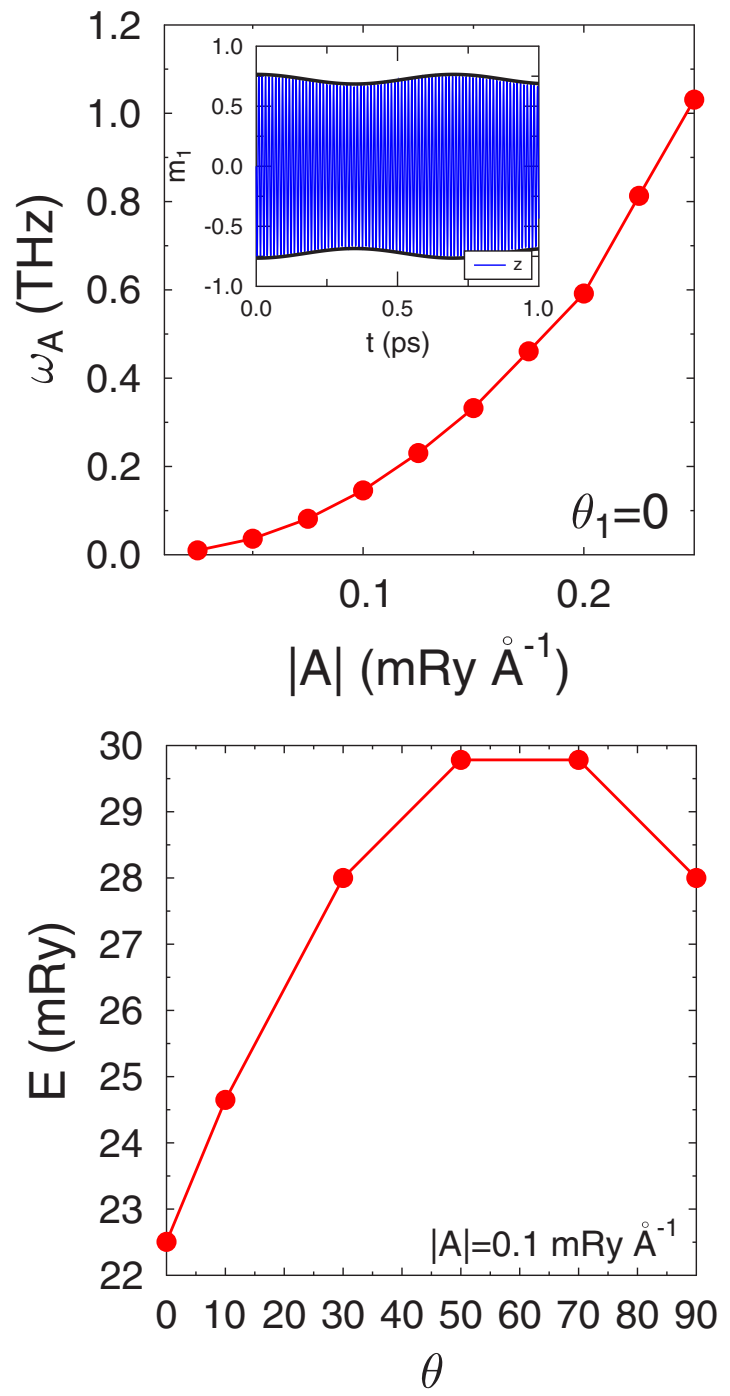

FIG. 7. Spin-lattice dynamics of trimer. (Top) Envelope frequency of spin oscillations as a function of $|\boldsymbol{A}|$. The inset shows the $z$ component of the trajectory of one magnetic moment in the trimer, and the definition of the envelope frequency (thick line). (Bottom) Total energy as a function of tilt angle of $A$.

lower in energy, at $3.2 \mathrm{THz}$, represents the lattice vibrations while magnetic fluctuations are responsible for the peak at 8.2 THz. In the case of a finite spin-lattice coupling, we find that for two of the considered angles $\left(\theta_{1}=0^{\circ}\right.$ and $\theta_{1}=$ $70^{\circ}$ ), a very fine splitting of the magnetic energy level is noticeable while a low-energy peak at $2.2 \mathrm{THz}$ occurs for the lattice vibrations. Interestingly, for the third choice of angle, $\theta_{1}=30^{\circ}$, the difference compared to the decoupled system is found to be minimal. Frequencies such as the ones shown in Fig. 6 are available through Raman spectroscopy, and an experimental detection of a spin-lattice coupling, via the split peaks in Fig. 6 would be interesting.

In our simulation, we varied both the strength $A$ and the angle $\theta_{1}$ of the exchange striction coupling. The strength $A$ affects the frequency $\omega_{A}$ of an enveloping oscillation on top of the spin precession frequency $\omega_{p}$ (see Fig. 7 upper panel and inset in upper panel): $\omega_{A}$ scales quadratically with the strength (a)

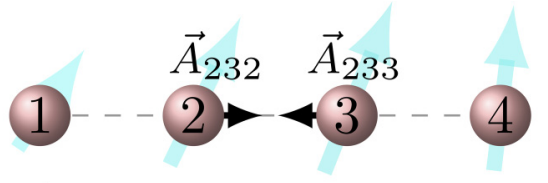

(b)

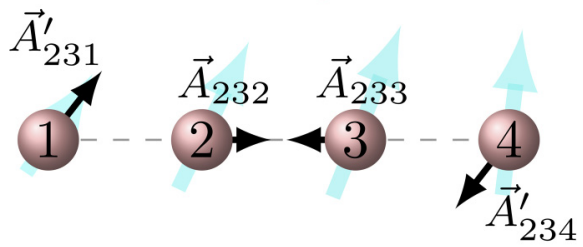

(c)

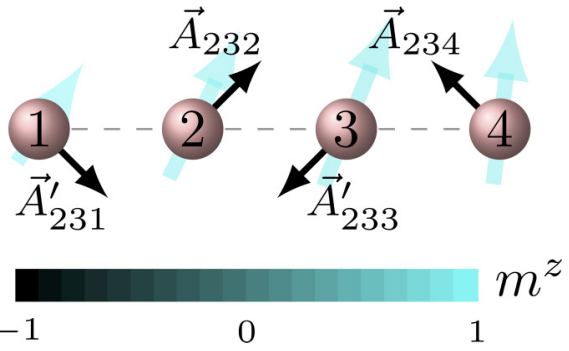

FIG. 8. Conceptual figure of the exchange striction coupling constants $\boldsymbol{A}_{23 k}, k=1,2,3,4$ (black arrows) in a tetramer. By mirror symmetry and to conserve the center of mass, $\boldsymbol{A}_{232}=-\boldsymbol{A}_{233}$ and $\boldsymbol{A}_{234}^{\prime}=-\boldsymbol{A}_{231}^{\prime}$ are allowed. The initial, random magnetic moment configuration in the tetramer is given by blue arrows, where the length and orientation is related to the in-plane component, and the color to the $z$ component of the moment (see color code on the bottom of the figure). The dotted line indicates the bond axis.

$A$ and is related to the difference between the frequencies caused by the exchange interaction term and the exchange striction term (see Fig. 6 green lines). Without energy dissipation, the magnetic energy is conserved, just as for the dimer case. The variations of the various energy contributions will be similar to the one in Fig. 2. The total energy increases linearly with the strength $A$, but oscillates with $\theta_{1}$, which is related to the fixed initial spin configuration (see Fig. 7 lower panel).

\section{Tetramer}

Although the trimer offers already rich phenomena, it addresses only nearest-neighbor couplings, which are symmetry related and not independent. Contrary to the trimer, the sites of a four-site system with periodic boundary condition [chain of atoms, see Fig. 8(a)], has both nearest neighbor (NN) and next-nearest neighbors (NNN). Consequently, it is possible to have finite couplings $\boldsymbol{A}_{132}=\boldsymbol{A}_{312}=-\boldsymbol{A}_{134}=\boldsymbol{A}_{314}$ also when $\boldsymbol{A}_{i j i} \| \mathbf{r}_{i j}$.

Here, we consider three different cases for the three-body interaction in the chain of four atoms. (1) Exchange striction vectors parallel to bonds between spin $i$ and $j$ [Fig. 8(a)]: $\boldsymbol{A}_{i j i} \| \mathbf{r}_{i j}, \boldsymbol{A}_{i j k(k \neq i, j)}=\mathbf{0}$. (2) Exchange striction vectors parallel to bonds between spin $i$ and $j$, but the second-nearestneighbor coupling is different from zero [Fig. 8(b)]: $\boldsymbol{A}_{i j i} \|$ $\mathbf{r}_{i j}, \boldsymbol{A}_{i j k(k \neq i, j)} \neq \mathbf{0}$. Thus the sum over the second-nearestneighbor couplings has to cancel: $\sum_{k \in \mathrm{NNN}} \boldsymbol{A}_{i j k}=0$. Furthermore, we enforce the mirror symmetry by setting $\boldsymbol{A}_{i j i}=$ $-\boldsymbol{A}_{i j j}$. (3) Exchange striction vectors not parallel to bonds for all indices [Fig. 8(c)]: $\boldsymbol{A}_{i j i} \nVdash \mathbf{r}_{i j}, \boldsymbol{A}_{i j k(k \neq i, j)} \neq \mathbf{0}$. We enforce 


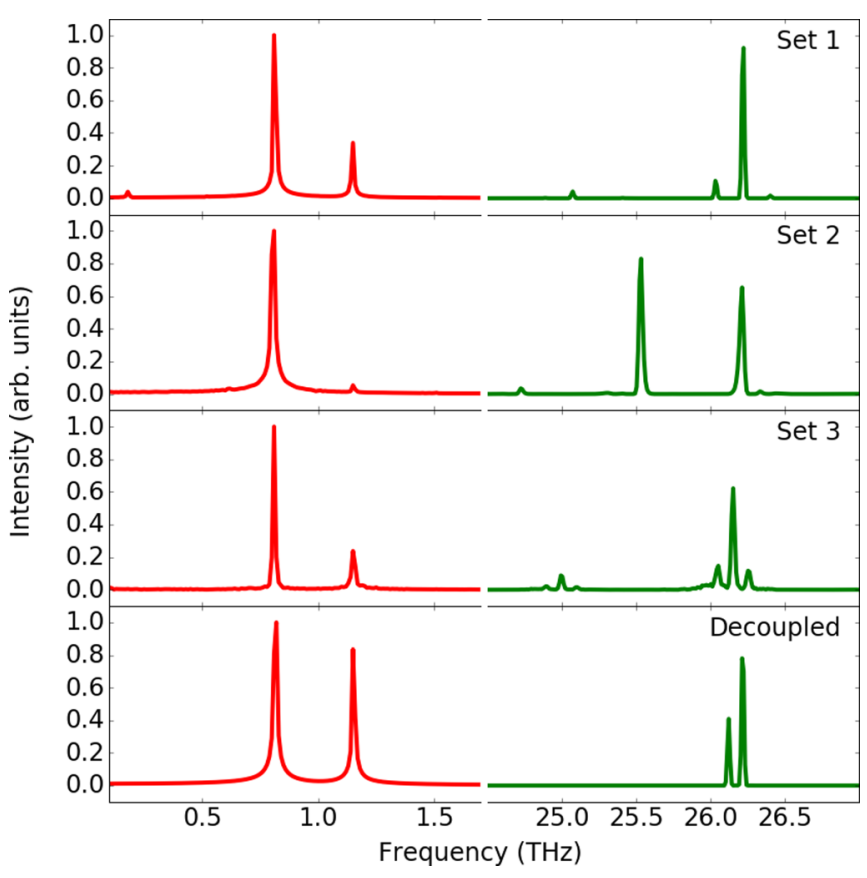

FIG. 9. Excitation spectra for lattice displacements (red/left lines) and magnetic oscillations (green/right lines) for the considered tetramer clusters. The top three panels show the excitations for the SLD simulations corresponding to the three different cases discussed in the text. The bottom panel corresponds to decoupled LD and SD excitations.

the sum rule [Eq. (11)] and also the mirror symmetry $\boldsymbol{A}_{i j i}=$ $-\boldsymbol{A}_{i j j}$.

For the Heisenberg exchange $J_{i j}$ and force constants $\Phi_{i j}^{\mu \nu}$, we include only nearest neighbor interactions of $J_{\mathrm{NN}}=$ $1 \mathrm{mRy} \mu_{\mathrm{B}}^{-2}$ and $\Phi_{12}^{x x}=-0.07 \mathrm{Ry} \AA^{-2}$. The atomic mass is put to 10 atomic mass units. To obtain the excitation spectra for these sets of tetramers, we perform microcanonical simulations for pre-equilibrated systems at a temperature of $10 \mathrm{~K}$. In Fig. 9, we show selected parts of the simulated excitation spectra for the three sets of tetramer configurations discussed above, together with results from a decoupled simulation where all $\boldsymbol{A}_{i j k}=0$. Starting with the decoupled results in the lower panel of Fig. 9 it is found that the lattice excitations are about one order of magnitude lower in energy compared to the magnetic excitations. For the magnetic excitations we find clear peaks at 26.2 and $52.5 \mathrm{THz}$ (data not shown for the highenergy peak), which correspond well with the eigenvalues of the decoupled spin Hamiltonian at zero temperature. The effect of finite spin-lattice couplings, shown in the top three panels in Fig. 9, is directly visible for the lattice vibrations where the spectral weight is shifted from the high energy peak. An effect of the spin-lattice coupling is also noticeable for the magnetic excitations, where the peak structure for the decoupled excitations changes. Here it is instead the lower energy peak that seems to shift towards lower energies. For set 3 , this shift is also accompanied with a broadening of the excitation peaks. For this set, we also notice what appears to be side bands of the excitations and we attribute these to the reduced symmetry of the Hamiltonian, to be discussed below.
In effective pair-interaction models, as used for instance in Refs. [106,107], only interactions between sites $i$ and $j$ are possible. This situation corresponds to the system depicted in Fig. 8(a) with its excitation spectra displayed in the top panel of Fig. 9, as well as the decoupled case with excitation spectra in the lowest panel of Fig. 9. Allowing for three-body interactions, as in the system shown in Figs. 8(b) and 8(c), lowers the symmetry of the interaction Hamiltonian, which in general allows for breaking of degenerate frequencies. For the results displayed in Fig. 9 (the two central panels) we have, for simplicity, set the NN and NNN three-body exchange striction to have the same strength, $1 \mathrm{mRy} \mu_{\mathrm{B}}^{-2} \AA^{-1}$. The resulting excitations do indeed show an increased number of modes, in particular for the magnetic modes. This is clear both concerning the position of the peaks, the number of peaks and the spectral weights that change significantly when including NNN three-body exchange couplings.

\section{B. Spin-lattice dynamics of bec $\mathrm{Fe}$}

In this section, we describe the Hamiltonian needed for coupled spin-lattice dynamics of bcc Fe. Not all terms described in Eq. (8) are needed for this case, and a simplified Hamiltonian appropriate for this system can be written as follows:

$$
\begin{aligned}
\mathcal{H}_{\mathrm{SLD}}= & -\frac{1}{2} \sum_{i j} J_{i j} \mathbf{m}_{i} \cdot \mathbf{m}_{j}-\frac{1}{2} \sum_{i j k} \mathbf{A}_{i j k} \cdot \mathbf{u}_{k}\left(\mathbf{m}_{i} \cdot \mathbf{m}_{j}\right) \\
& +\frac{1}{2} \sum_{k l} \Phi_{k l}^{\mu \nu} u_{k}^{\mu} u_{l}^{v}+\frac{1}{2} \sum_{k} M_{k} v_{k}^{\mu} v_{k}^{\mu} .
\end{aligned}
$$

Here, the first term is the Heisenberg term with scalar exchange parameters $J_{i j}$, and the second term describes the exchange striction, Eq. (9), with strength given by the exchangestriction vectors $\boldsymbol{A}_{i j k}$. The two terms on the second line are the terms describing the lattice dynamics, Eq. (2).

\section{Coupling constants for bcc Fe}

In this section. we discuss the coupling parameters that we obtain from DFT, namely, Heisenberg exchange $J_{i j}$, force constants $\Phi_{i j}$, and exchange striction $\boldsymbol{A}_{i j k}$.

The force constants are obtained from supercell calculations and are related by point-group symmetry [108-111]. Hence, we list only the irreducible values in Table I. In the table, $k=1$ denotes the nearest-neighbor shell, $k=2$ the next-nearest-neighbor shell, and so forth. The indices $\mu$ and $v$ stand for Cartesian coordinates.

The Heisenberg exchange for bcc Fe is well studied and our calculations agree well with previous work [78,95,112-114]. The $J$ 's are isotropic, long ranged, oscillating with decay typically as $r^{-3}$. The calculations reveal that $J \approx 0$ at $10 \mathrm{~nm}$. In Fig. 10(a), the exchange energy is plotted against distance $r_{i j}$. The nearest-neighbor interaction is about $J_{\mathrm{NN}} \mu_{\mathrm{Fe}}^{2}=1 \mathrm{mRy}$ where we use a magnetic moment of $m_{\mathrm{Fe}}=2.23 \mu_{\mathrm{B}}$. With also the second nearest neighbor-interaction positive, bcc Fe is ferromagnetic. 
TABLE I. The elements $\Phi_{0 k}^{\mu v}$ of the force-constant matrix in bcc Fe.

\begin{tabular}{|c|c|c|c|}
\hline$k$ & $\begin{array}{c}\mu \nu \\
\text { irreducible }\end{array}$ & $\begin{array}{c}\mu \nu \\
\text { reducible }\end{array}$ & $\Phi_{0 k}^{\mu v}\left(\mathrm{mRy} \AA^{-2}\right)$ \\
\hline 1 & $\begin{array}{l}x x \\
x y\end{array}$ & $\begin{array}{c}x x, y y, z z \\
x y, x z, z x, y z, z x, z y\end{array}$ & $\begin{array}{l}-4360 \\
-2670\end{array}$ \\
\hline 2 & $x x$ & $x x$ & -4850 \\
\hline 2 & $\begin{array}{l}y y \\
x y\end{array}$ & $\begin{array}{c}y y, z z \\
x y, x z, z x, y z, z x, z y\end{array}$ & $\begin{array}{r}-2230 \\
0\end{array}$ \\
\hline 3 & $\begin{array}{l}x x \\
x y \\
z z \\
x y\end{array}$ & $\begin{array}{c}x x, y y \\
x y, y x \\
z z \\
x z, z x, y z, z y\end{array}$ & $\begin{array}{r}-459 \\
-209 \\
403 \\
0\end{array}$ \\
\hline 4 & $\begin{array}{l}x x \\
x y \\
y y \\
y z\end{array}$ & $\begin{array}{c}x x \\
x y, x z, y x, x y \\
y y, z z \\
y z, z y\end{array}$ & $\begin{array}{r}55 \\
-48 \\
-126 \\
-251\end{array}$ \\
\hline 5 & $\begin{array}{l}x x \\
x y\end{array}$ & $\begin{array}{c}x x, y y, z z \\
x y, x z, z x, y z, z x, z y\end{array}$ & $\begin{array}{r}122 \\
-169\end{array}$ \\
\hline
\end{tabular}

From our first-principles calculations, we obtained magnitudes $|\boldsymbol{A}|=A$ and directions $\boldsymbol{e}^{A}$ of the spin-lattice coupling. We distinguish between the set of couplings $\{\boldsymbol{A}\}$ in a subset where (i) the site $k$ is equal to site $i$ or $j$, e.g., $\boldsymbol{A}_{i j i}$, and where (ii) $k \neq i, j$. Case (i) refers to the couplings between two magnetic moments, where the lattice displacement occurs on one of these two magnetic sites. This kind of couplings were used in Refs. $[54,71,85]$. Our calculations based on density functional theory demonstrate that couplings at $k$ where $k \neq$ $i, j$ are also important. This will be discussed in more detail below, in connection to Fig. 10(b).

We note that the general notation of the exchange striction coupling is $\boldsymbol{A}_{i j k}$. This coupling indicates the modification of the exchange interaction between site $i$ to $j$ (of distance $\boldsymbol{r}_{i j}$ ) with respect to a displacement of an atom $k$ (separated with distance $\boldsymbol{r}_{i k}$ from site $i$ ). For simplicity, in the discussion below we focus on the couplings where the distances $\boldsymbol{r}_{i k}$ are between nearest neighbors $(\mathrm{NN})$ and next-nearest neighbors $(\mathrm{NNN})$ only, while values for $\boldsymbol{r}_{i j}$ can be longer ranged.

We start our discussion with case (i) and present DFT calculations in Fig. 10(b). We show in the figure values of $A$ for five values of $\boldsymbol{r}_{i j}$. Note from the figure that the value of $A$ decays slowly with distance between spins, starting at a value of $A \mu_{\mathrm{Fe}}^{2}=0.64 \mathrm{mRy} \AA^{-1}$ and ending at a value close to zero for the longest distance. In general, the magnitude of $A_{i j i}$ does not decay as quickly with distance $\boldsymbol{r}_{i j}$, as the exchange interactions do [in Fig. 10(a)]. Further, an outcome of our density functional theory simulations is that the direction of $A_{i j i}$ align along the distance vector $\boldsymbol{r}_{i j}$. This implies that for this set of couplings the Heisenberg exchange $J_{i j}$ can be assumed to be a function only of the distance $\boldsymbol{r}_{i j}$, because $\nabla_{i} J_{i j}\left(\boldsymbol{r}_{i j}\right)=\partial J_{i j} / \partial r_{i j} \boldsymbol{e}_{i j}^{r}$. It also implies that the magnitude of the $\boldsymbol{A}_{i j i}$ coupling is isotropic, and that the sum rule in Eq. (11) simplifies to $\boldsymbol{A}_{i j i}=-\boldsymbol{A}_{i j j}$.

In case of (ii) $(k \neq i, j)$, the strength of the exchange striction coupling depends on two vectors $\boldsymbol{r}_{i j}$ and $\boldsymbol{r}_{i k}$. Note that for a fixed value of $\boldsymbol{r}_{i j}$ there could be several values

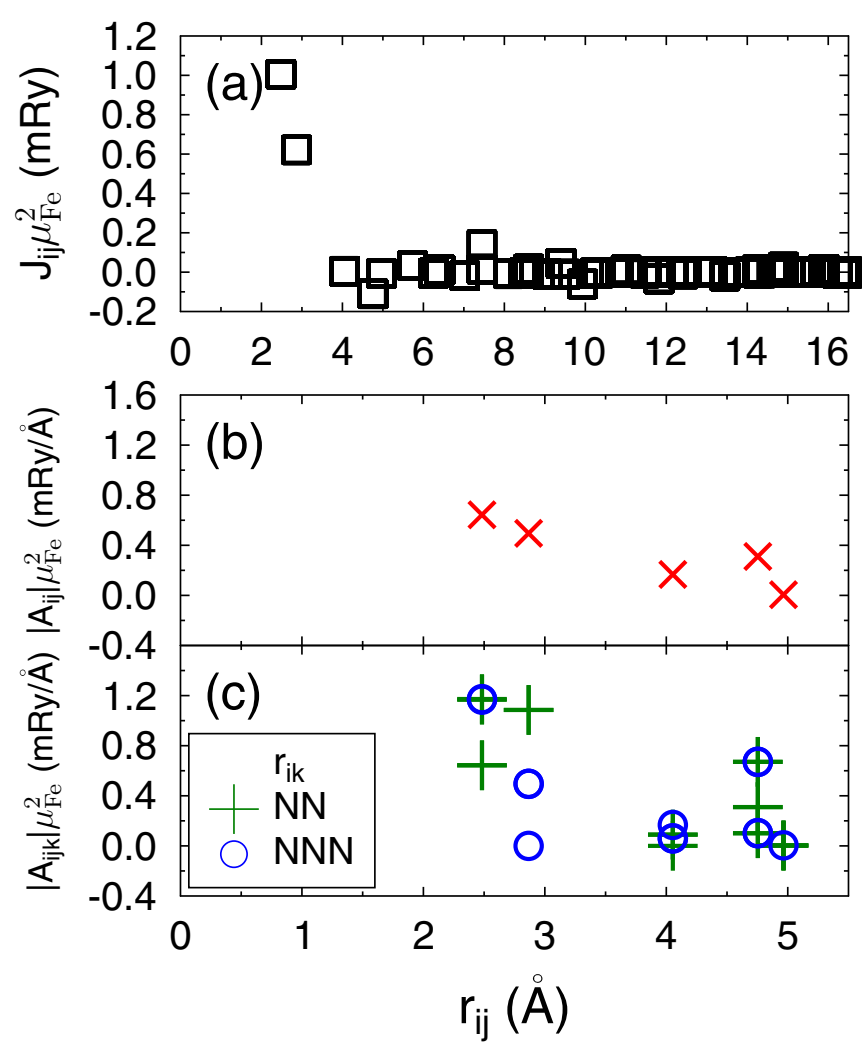

FIG. 10. (a) The Heisenberg exchange coupling $J_{i j}$ as a function of the distance $r_{i j}$. (b) Absolute value of the exchange striction $\boldsymbol{A}_{i j k}$ as a function of the distance $r_{i j}$, for on-site contribution $\left(r_{i k}=0\right.$; red cross). (c) Absolute value of the exchange striction $\boldsymbol{A}_{i j k}$ as a function of the distance $r_{i j}$, for $r_{i k}=\mathrm{NN}$ contribution (green plus in lower panel) and $r_{i k}=$ NNN contribution (blue circle in lower panel).

of $\boldsymbol{r}_{i k}$, which means that in Fig. 10(c) there are in general several values of $A$ for the same $\boldsymbol{r}_{i j}$ value. For the cases when $\boldsymbol{r}_{i j}$ is parallel/antiparallel to $\boldsymbol{r}_{i k}$, we find that the values of $A$ differ from the exchange striction couplings for which $\boldsymbol{r}_{i j}$ and $\boldsymbol{r}_{i k}$ are not collinear. We emphasize that the degeneracy of certain points in Fig. 10(c) mirrors the symmetry property $A_{i j k}=A_{j i k}$. We further note from Fig. 10(c) that for fixed value of $\boldsymbol{r}_{i k}$ the general trend for $A$ is to decay as a function of increasing value of $\boldsymbol{r}_{i j}$. Both for nearest-neighbor and nextnearest-neighbor distances $\boldsymbol{r}_{i k}$, these $(k \neq i, j)$ couplings give significant contributions to the exchange striction. The decay of $A$ with distance $\boldsymbol{r}_{i j}$ is rather similar in Figs. 10(b) and 10(c). Note also that for general $\boldsymbol{A}_{i j k}$ the orientation $\boldsymbol{e}^{A}$ must not necessarily be along the distance vector $\boldsymbol{r}_{i k}$.

\section{Thermodynamic properties of bcc Fe}

In order to investigate how the presence of exchange striction affects the magnetization order parameter at finite temperature, we have performed Langevin dynamics, spinlattice dynamics (SLD), uncoupled spin dynamics (SD), and lattice dynamics (LD) simulations in the temperature range 0 to $1500 \mathrm{~K}$ for simulation cells with size $N \times N \times N$ and periodic boundary conditions. $N_{t}=1 \times 10^{6}$ time steps of $d t=10^{-16} \mathrm{~s}$ were used in combination with the damping parameters $\alpha=1.00$ and $v=10^{-14} \mathrm{~s}^{-1}$. For low and 

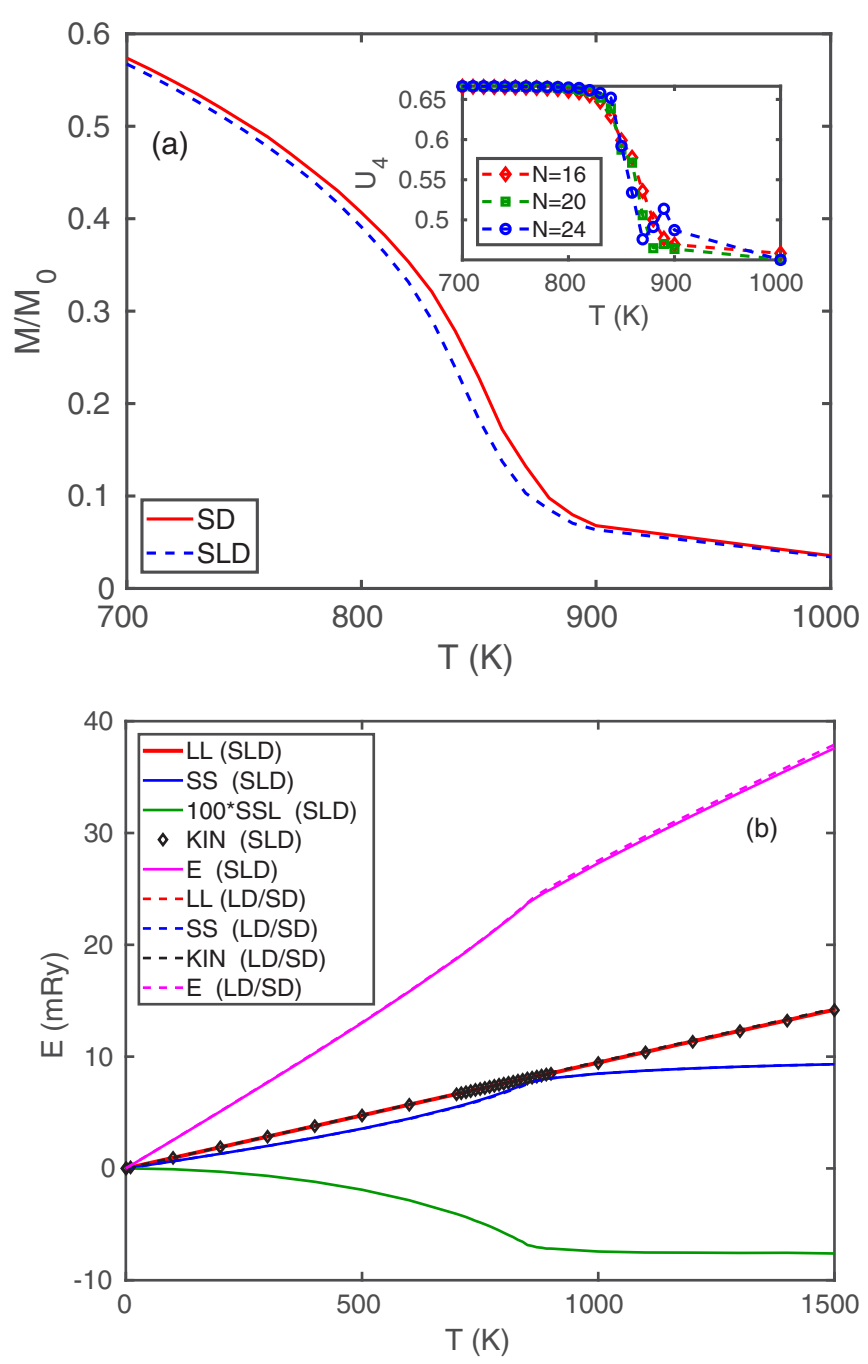

FIG. 11. (a) The magnetic order parameter $M(T) / M(T=0)$ sampled in SLD and in SD simulation for a $N=20$ simulation cell. The inset shows the crossing of the $U_{4}$ Binder cumulant for cell sizes $N=16$, 20, and 24 SLD simulations. (b) Energies of the spin-lattice dynamics Hamiltonian as a function of temperature.

high temperatures, the magnetic order parameters deviate negligibly when comparing SLD and SD data, but, as shown in Fig. 11(a), in the vicinity of the phase transition temperature the order parameter takes a lower value in the SLD simulation than in the SD simulation. The inset shows the crossing of the $U_{4}$ Binder cumulant for cell sizes $N=16,20$, and 24 SLD simulations from which a critical temperature $T_{c}=850 \mathrm{~K}$ can be read out. The energies of the different terms in the spinlattice Hamiltonian are shown as a function of temperature in Fig. 11(b). We can observe that the difference in the energies for SLD simulation compared to energies for the uncoupled LD and SD simulation are smaller than the line-size in the graph, apart from, naturally, the exchange striction energy (SSL), which is identically zero in the uncoupled simulation and finite for the coupled system. The SLL energy change is small compared to the total energy (E). The harmonic lattice potential (LL) and the kinetic energy (KIN) coincide as expected given the equipartition theorem and are linear in
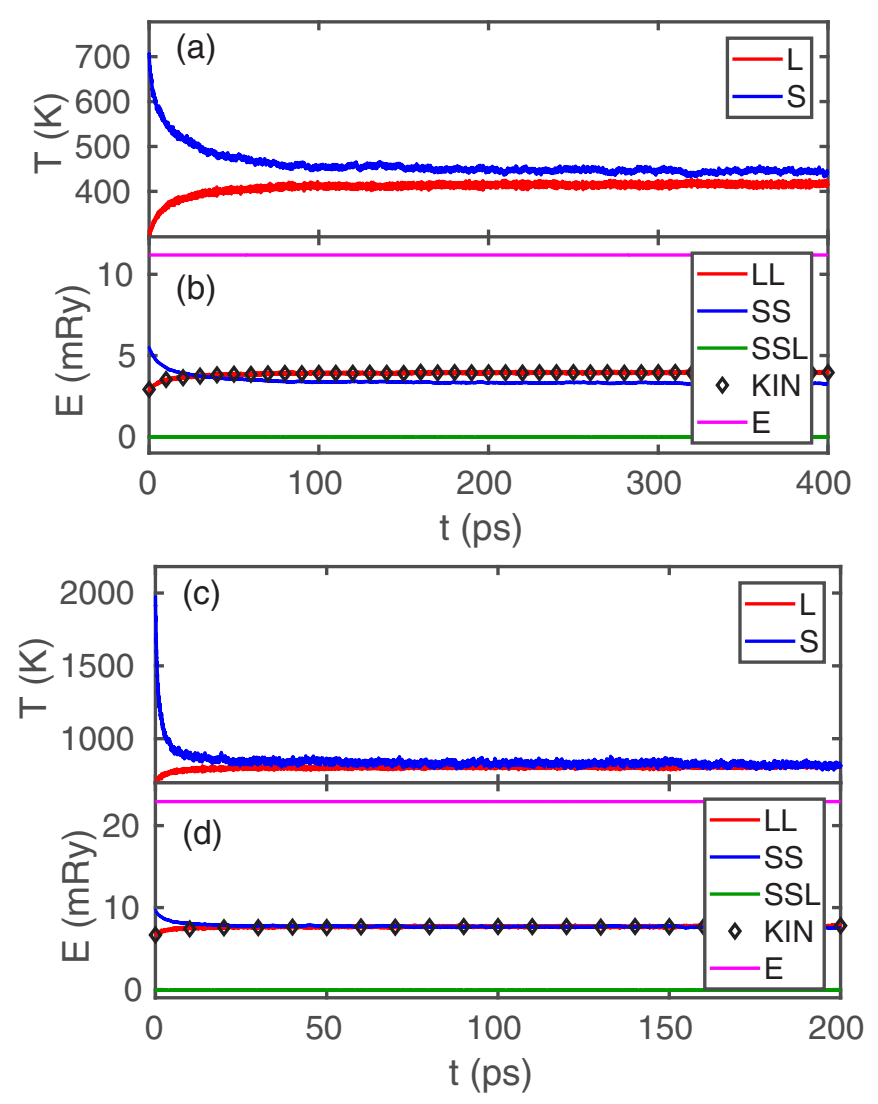

FIG. 12. Exchange-striction mediated relaxation of the temperature and energy of spin and lattice subsystem in microcanonical evolution for two sets of initial temperatures of the subsystems. In (a) and (b), the initial spin temperature was $700 \mathrm{~K}$ and the initial lattice temperature was $300 \mathrm{~K}$. In (c) and (d), the initial spin temperature was $2000 \mathrm{~K}$ and the initial lattice temperature was $700 \mathrm{~K}$. At $t=0$, the spin and lattice systems are each in thermal equilibrium with these heat baths. At $t>0$, the system evolves with Hamiltonian dynamics, with the total energy conserved but redistributed between the available degrees of freedom.

temperature. Unlike the lattice energies, the magnetic energy (SS) has an upper bound and flattens out above the phase transition temperature and is taking a concave shape with values smaller than the lattice energy at lower temperature.

Figure 12 shows exchange striction mediated relaxation of the temperature and energy of spin and lattice subsystem in microcanonical evolution of the $N_{\text {atoms }}=8000$ cell with edge length $N=20$, for undamped simulations. We calculate the temperature of the lattice subsystem from the kinetic energy, while for the spin system, the temperature is obtained using the method by Ma et al. [115], At $t=0 \mathrm{ps,} \mathrm{the} \mathrm{spin}$ system and lattice system are in thermal equilibrium with heat baths at different temperatures. At $t>0$, the system evolves in Hamiltonian dynamics simulated with the fixedpoint scheme for implicit mid-point method (see Appendix) using a time step $d t=10^{-16} \mathrm{~s}$. The total energy is conserved but it is redistributed between the degrees of freedom. For initial conditions $T_{S}=700 \mathrm{~K}$ and $T_{L}=300 \mathrm{~K}$ are shown the time trajectories of temperature in Fig. 12(a) and energies in Fig. 12(b). As expected from the equipartition theorem, the 
lattice harmonic potential energy and kinetic energy take the same values. Complete equilibration of the spin and lattice subsystem to the same temperature does not happen during the displayed time interval of $400 \mathrm{ps}$. We attribute this incomplete relaxation to a kinematic constraint due to not only total energy, but also the total spin angular momentum, being constants of motion in these microcanonical simulations. At $t=0$, the average magnetization is $M \approx 1.3 \mu_{B}$ and as no net torque act on the spin system, energy can be transferred from the spin system to the lattice only in dynamics in which the magnetization is preserved. The relaxation of spin and lattice exponential temperatures towards an almost common temperature is exponential decay. Fitting to single exponential functions $T=a \exp (-t / \tau)+b$, we obtain a relaxation time of $\tau_{L} \approx \tau_{S} \approx 15$ ps. The situation is different in Figs. 12(c) and $12(\mathrm{~d})$ where the initial condition is $T_{S}=2000 \mathrm{~K}$ and $T_{L}=$ $700 \mathrm{~K}$, and relaxation to a common temperature $T_{S}=T_{L} \approx$ $800 \mathrm{~K}$ occur, a process which is possible given that the system is paramagnetic at $T_{S}=2000 \mathrm{~K}$, and that the net equilibrium magnetization is very small at $T_{S}=800 \mathrm{~K}$. The relaxation to this temperature proceeds with a relaxation time $\tau_{L} \approx \tau_{S} \approx 2$ ps, faster than for Figs. 12(a) and 12(b). We note that at this temperature the harmonic potential, kinetic energy, and Heisenberg exchange coincide, in agreement with the thermal equilibrium data in Fig. 11. Finally, we note that in contrast to the presently discussed results for a ferromagnetic system, antiferromagnetic dynamics allow for relaxation without an angular momentum bottleneck, see, e.g., the relaxation in Hamiltonian dynamics reported in Ref. [63].

\section{3. bcc Fe magnon and phonon dispersions}

The simulations we have performed to sample the dynamic structure factor for bcc Fe were divided into two parts or stages: (i) equilibration stage with Langevin dynamics simulated with the combined velocity-Verlet and SIB solver algorithm, as described in Appendix, subdivided into phases with first a longer time step $\left(d t=10^{-15} \mathrm{~s}\right)$ and high damping, followed by gradually shorter time steps and lower damping. In the fourth and final phase, we used $N_{t}=10^{4}$ time steps of $d t=10^{-16} \mathrm{~s}$ and the damping parameters $\alpha=0.01$ and $v=10^{-14} \mathrm{~s}^{-1}$. (ii) Measurement stage consisting of Hamiltonian evolution of the system over $N_{t}=2 \times 10^{5}$ time steps of $d t=5 \times 10^{-16} \mathrm{~s}$ with the fixed-point iteration implicit midpoint method, see Appendix. The sampling step $t_{\mathrm{samp}}=$ $5 \times 10^{-15} \mathrm{~s}$ for the correlation functions defined in Eqs. (17)(19) is used for a sampling window of $t_{\text {win }}=5 \times 10^{-11} \mathrm{~s}$ and combined with averaging of the correlations by moving the time window over $t_{0}=\left(0,5,10, \ldots, 5 \times 10^{4}\right) \times 10^{-15} \mathrm{~s}$. The corresponding frequency range for the dynamic structure factors in Eqs. (20)-(22) is $\omega /(2 \pi)=[0.02,0.04, \ldots, 200]$ $\mathrm{THz}(0.0827$ to $827 \mathrm{meV})$.

In order to investigate the impact of the exchange striction on the magnon and phonon spectra at finite temperature, we pursued both spin-lattice dynamics simulations and uncoupled spin dynamics and lattice dynamics simulations. In the very detailed investigations by Perera et al. [59] of magnon and phonon spectra of the Dudarev-Derlet potential $[55,56]$ for bcc Fe, the ratio $\left(\omega_{\mathrm{SLD}}(Q)-\omega_{\mathrm{SD}}(Q)\right) / \omega_{\mathrm{SD}}(Q)$ was used to analyze the temperature-dependent influence

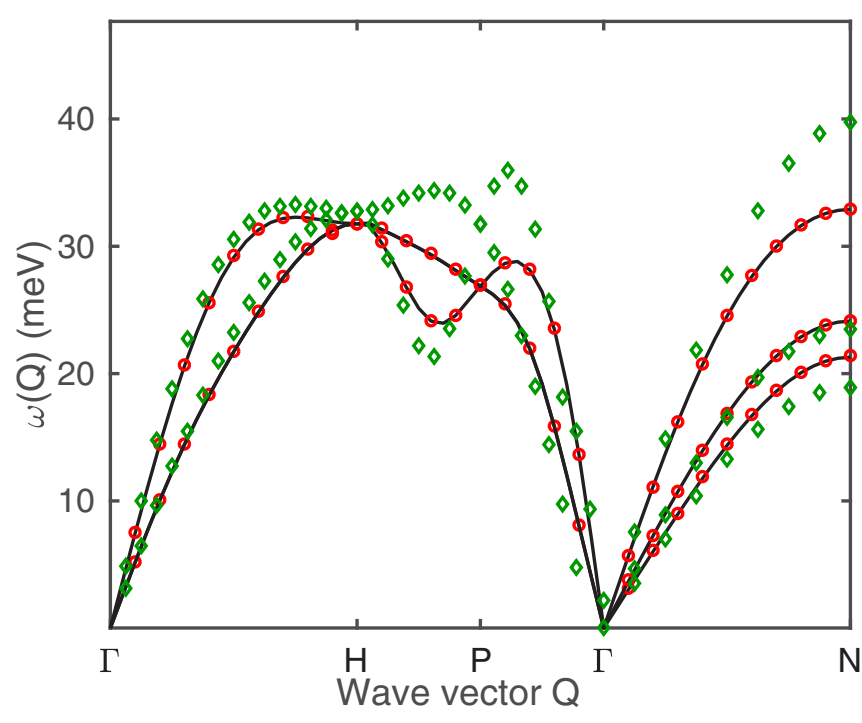

FIG. 13. The $T=0 \mathrm{~K}$ adiabatic phonon dispersion $\omega(\mathbf{Q})$ (black curve) calculated using Eq. (26), and the peaks of the displacementdisplacement dynamic structure factor $S_{(\mathbf{u})}^{x x}(\mathbf{Q}, \omega)+S_{(\mathbf{u})}^{y y}(\mathbf{Q}, \omega)+$ $S_{(\mathbf{u})}^{z z}(\mathbf{Q}, \omega)$ (red symbols) sampled in SLD simulations of bcc Fe at $T=300 \mathrm{~K}$ using a $20 \times 20 \times 20$ supercell with periodic boundary conditions. The $T=300 \mathrm{~K}$ dispersions from SLD simulations in Ref. [59] (green diamonds) are shown for comparison.

of exchange striction on magnon dispersion, and similarly, the ratio $\left(\omega_{\mathrm{SLD}}(Q)-\omega_{\mathrm{LD}}(Q)\right) / \omega_{\mathrm{LD}}(Q)$ was defined for the phonon dispersions. We will make use of the same ratios in analyzing the results of the present work.

In Fig. 13, we show the sum of diagonal components of the displacement-displacement dynamic structure factor, $S_{(\mathbf{u})}^{x x}(\mathbf{Q}, \omega)+S_{(\mathbf{u})}^{y y}(\mathbf{Q}, \omega)+S_{(\mathbf{u})}^{z z}(\mathbf{Q}, \omega)$, sampled in SLD simulation at $T=300 \mathrm{~K}$ (red circles), as well as the $T=0$ $\mathrm{K}$ adiabatic phonon dispersion $\omega(\mathbf{Q})$ obtained from use of Eq. (26) (black curve). As seen by comparing the dispersion at $T=0$ and $300 \mathrm{~K}$, our simulations do not predict any significant softening of the phonon dispersion with temperature. In the same figure, we also show the corresonding SLD phonon spectrum data taken from Ref. [59] (green diamonds), for comparison. Overall, the spectra are quite similar. Along $\Gamma-\mathrm{H}$, the simulation results from Ref. [59] and our simulation agree quantitatively. Along the other paths, however, there are visible discrepancies, with the phonon frequencies in the simulation results from Ref. [59] displaying an overall higher dependence on the $\boldsymbol{Q}$ wave vector. In addition, along the H-P path in the BZ the simulation results from Ref. [59] show an additional structure compared to our simulations. In our modeling we use five coordination shells for the atomic force constants (see Table I), the same number of shells as was used for analyzing phonon dispersions measured in inelastic neutron scattering experiments by Minkiewicz et al. [116] and by Klotz and Braden [117], and we note that the shape of our phonon dispersion is similar to Minkiewicz' et al. and to Klotz and Braden's results. The Hamiltonian in Eq. (36) does not include any anharmonic lattice potential contributions, and we did in the pure lattice dynamics (LD) simulations not observe any change in the phonon dispersion with temperature. 

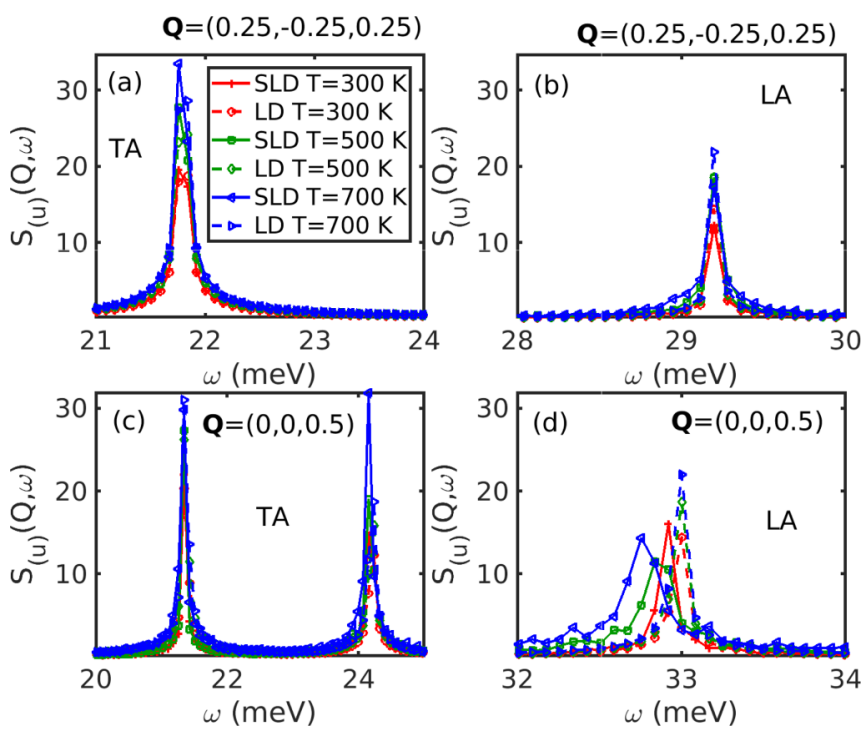

FIG. 14. The peaks of the displacement-displacement dynamic structure factor $S_{(\mathbf{u})}^{x x}(\mathbf{Q}, \omega)+S_{(\mathbf{u})}^{y y}(\mathbf{Q}, \omega)+S_{(\mathbf{u})}^{z z}(\mathbf{Q}, \omega)$ for selected points in the Brillouin zone, sampled in SLD and LD simulations of bec Fe at $T=300,500$, and $700 \mathrm{~K}$. For $\mathbf{Q}=(0.25,-0.25,0.25)$, i.e., halfway between $\Gamma$ and $H$ are shown the peaks for the transverse acoustic (a) and longitudinal acoustic mode (b). For the $N$ point, $\mathbf{Q}=(0,0,0.5)$, the peak of the transverse acoustic modes are shown in (c), and the peak of the longitudinal acoustic mode in (d).

In Fig. 14, we show the dynamic structure factor as a function of frequency for selected $\boldsymbol{Q}$ points, and for different temperature. Considering first the point $\mathbf{Q}=(0.25,-0.25,0.25)$, i.e., halfway between $\Gamma$ and $\mathrm{H}$, we observe that, within the resolution of the present Fourier mesh, there is no visible softening or broadening of the phonon dispersions with temperature, neither for the transverse acoustic peak [Fig. 14(a)], or for the longitudinal acoustic mode [Fig. 14(b)], however a slight increase of the peak intensity of $S_{(\mathbf{u})}^{x x}(\mathbf{Q}, \omega)+S_{(\mathbf{u})}^{y y}(\mathbf{Q}, \omega)+$ $S_{(\mathbf{u})}^{z z}(\mathbf{Q}, \omega)$ with temperature, in agreement with the expectation that more phonons are excited at higher temperatures. In addition, the graphs from the SLD and the LD simulations are very close to each other. Also the peaks of two transverse acoustic modes at the $\mathrm{N}$ point displayed in Fig. 14(c) increase with temperature, but do not change seemingly with with exchange striction. More intriguing is the situation for the $\mathrm{N}$ point peak of the longitudinal acoustic branch shown in (d). Here the influence of exchange striction can be seen to be twofold, it leads both to a change of the dispersion, and to a broadening of the resonance.

In order to analyze how much exchange striction influences, the phonon dispersions along high-symmetry directions in the Brillouin zone, we calculate the relative change using the expression $\left(\omega_{\mathrm{SLD}}(Q)-\omega_{\mathrm{LD}}(Q)\right) / \omega_{\mathrm{LD}}(Q)$, which we display for temperatures $T=300,500$, and $700 \mathrm{~K}$ in Fig. 15 . For both the longitudinal and the transversal branches, the largest relative change of the dispersion occur at the $\mathrm{H}$ point, but there is a minimum of the change half way between $\Gamma-\mathrm{H}$, at $\mathbf{Q}=(0.25,-0.25,0.25)$. The modulation of the dispersion is small along the $\mathrm{P}-\Gamma$ path and is of maximum $-0.5 \%$. For the $\Gamma-N$ path, there is a minimum for the modulation occurring

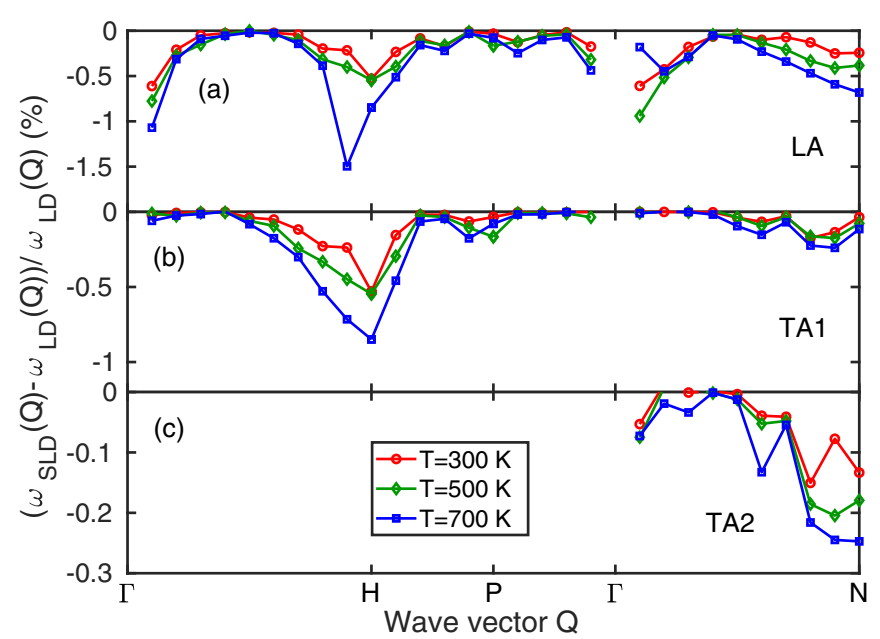

FIG. 15. The dispersions fitted from the displacementdisplacement dynamic structure factor $S_{(\mathbf{u})}^{x x}(\mathbf{Q}, \omega)+S_{(\mathbf{u})}^{y y}(\mathbf{Q}, \omega)+$ $S_{(\mathbf{u})}^{z z}(\mathbf{Q}, \omega)$ sampled in SLD simulations and in LD simulations of bcc $\mathrm{Fe}$ at different temperatures using a $20 \times 20 \times 20$ supercell with periodic boundary conditions. The panels show the ratio $\left(\omega(\mathbf{Q})_{\mathrm{SLD}}-\omega(\mathbf{Q})_{\mathrm{LD}}\right) / \omega(\mathbf{Q})_{\mathrm{LD}}$, at $T=300$ (red), 500 (green), and $700 \mathrm{~K}$ (blue) for the (a) longitudinal acoustic (LA), (b) transversal acoustic (TA), and for the $\Gamma-N$ path, (c) second transversal acoustic branch (TA2). Note that different vertical scales are used in (a), (b), and (c).

in the vicinity of the $\mathbf{Q}=(0.00,0.00,0.25)$ point. At the $N$ point, the softening of the dispersion can be seen to be larger for the one transverse acoustic branch with the higher frequency [panel (c)] than for the other transverse branch [panel (b)] for which the modulation of the frequency is less than $0.2 \%$ (cf. Fig 14). We note that both for the longitudinal and the transversal branches, and at all temperatures, can we observe a softening in the phonon dispersions when exchange striction is present, but no tendency to hardening to higher frequencies. This trend is consistent with the results shown in Fig. 3 in Körmann et al. in Ref. [21], but in contrast with Perera et al. in Ref. [59] who reported softening or hardening of the phonons for different paths of the Brillouin zone due to exchange striction.

Similarly, in Fig. 16, we display the $x x$ component of the spin-spin dynamic structure factor $S_{(\boldsymbol{m})}(\mathbf{Q}, \omega)$ (blue dashed line for the SLD simulation and red line for the SD simulation). The $T=0 \mathrm{~K}$ adiabatic magnon dispersion $\omega(\mathbf{Q})$ (black line) calculated using Eq. (24) is also shown. As a consequence of the RKKY type oscillation of the exchange interaction, Kohn anomalies [118] can be seen in the dispersions, as discussed in earlier DFT calculations for bcc Fe [78,112]. The insets of Fig. 16 show the shape of the Lorentzian functions at the $\mathrm{H}$ and $\mathrm{N}$ points for SLD and SD simulations, respectively. Comparing the shapes of these Lorentzian functions reveals that the presence of exchange striction in the SLD simulation causes a broadening of the resonance peaks as compared to the SD simulation. Also here, we can compare our results with data from Ref. [59], (shown as green diamonds in the figure). Along some of the paths in $\boldsymbol{Q}$ space, the agreement is excellent, for instance along $P-\Gamma$ and $\Gamma-N$. However, due to 


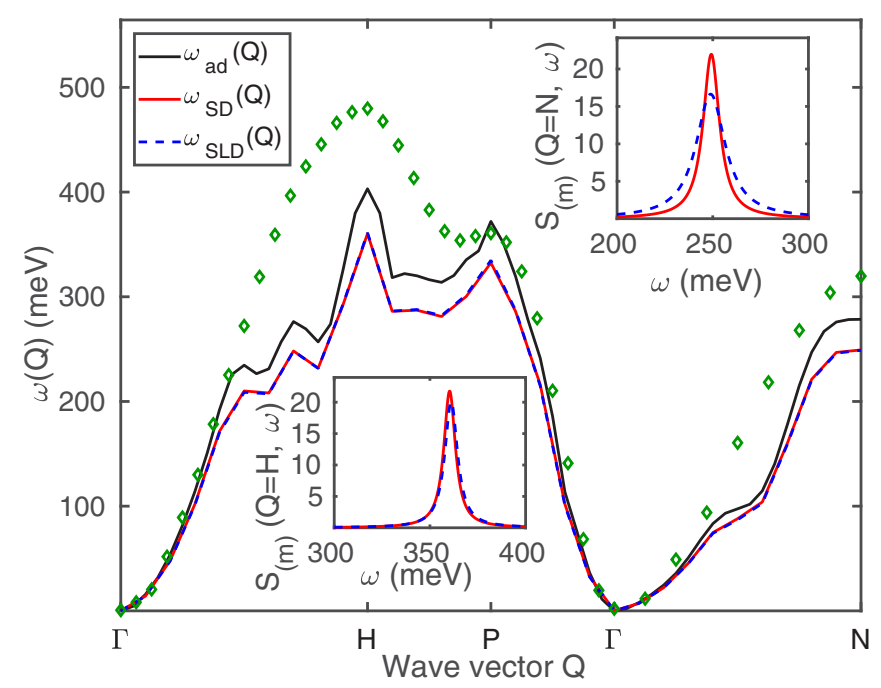

FIG. 16. The $T=0 \mathrm{~K}$ adiabatic magnon dispersion $\omega(\mathbf{Q})$ (black) calculated using Eq. (24), and the peak of the $x x$ component of the spin-spin dynamic structure factor $S_{(\mathbf{m})}(\mathbf{Q}, \omega)$ sampled in SLD (blue) and SD (red) simulations of bec Fe at $T=300 \mathrm{~K}$ using a $20 \times 20 \times 20$ supercell with periodic boundary conditions. The $T=300 \mathrm{~K}$ dispersions from SLD simulations in Ref. [59] (green diamonds) are shown for comparison. The insets show the shape of the Lorentzian functions at the $H$ and $N$ points for SLD and SD simulations, respectively.

the Kohn anomaly associated with the RKKY type oscillations of the exchange, along the paths $\Gamma-H$ and $H-P$ our spectra show a number of small peaks, that do not show up in the simulation results from Ref. [59] where there is instead a smoother, broader structure in this region. Also, in this part of the dispersion the two calculations differ significantly as regards the computed magnon energy. The discrepancy is largest around the $H$ point, where our computed magnon energy is around $380 \mathrm{meV}$, whereas in Ref. [59], it is about $480 \mathrm{meV}$, which is due to weaker exchange interactions in our calculations compared to those of Ref. [59].

It is interesting to investigate in more detail how the magnon spectrum depends on temperature. In Fig. 17(a), we show the dispersion fitted from the spin-spin dynamic structure factor at several different temperature. The decrease in the Heisenberg exchange field with increasing temperature, associated with the thermal fluctuations and the reduction of the magnetic order parameter, exert a much stronger handle on the magnon dispersion than the presence of exchange striction do. At each temperature $T=300,500$, and $700 \mathrm{~K}$ the dispersions obtained from SLD and SD simulations differ only slightly, however the softening with temperature is pronounced. In our present model for bcc Fe increasing temperature always leads to lower dispersion. In analog with how we analyzed the influence of exchange striction on the phonon dispersions, we do in Fig. 17(b) show results for the ratio $\left(\omega_{\mathrm{SLD}}(Q)-\omega_{\mathrm{SD}}(Q)\right) / \omega_{\mathrm{SD}}(Q)$. The statistics of the data obtained in our current finite temperature simulations is limited, in particular for the $T=700 \mathrm{~K}$ data, and in the following we concentrate on the $T=300$ and $500 \mathrm{~K}$ data. For the $\Gamma-H$ path, a softening of the magnon dispersion can be seen for small wave vectors, with the relative change being the
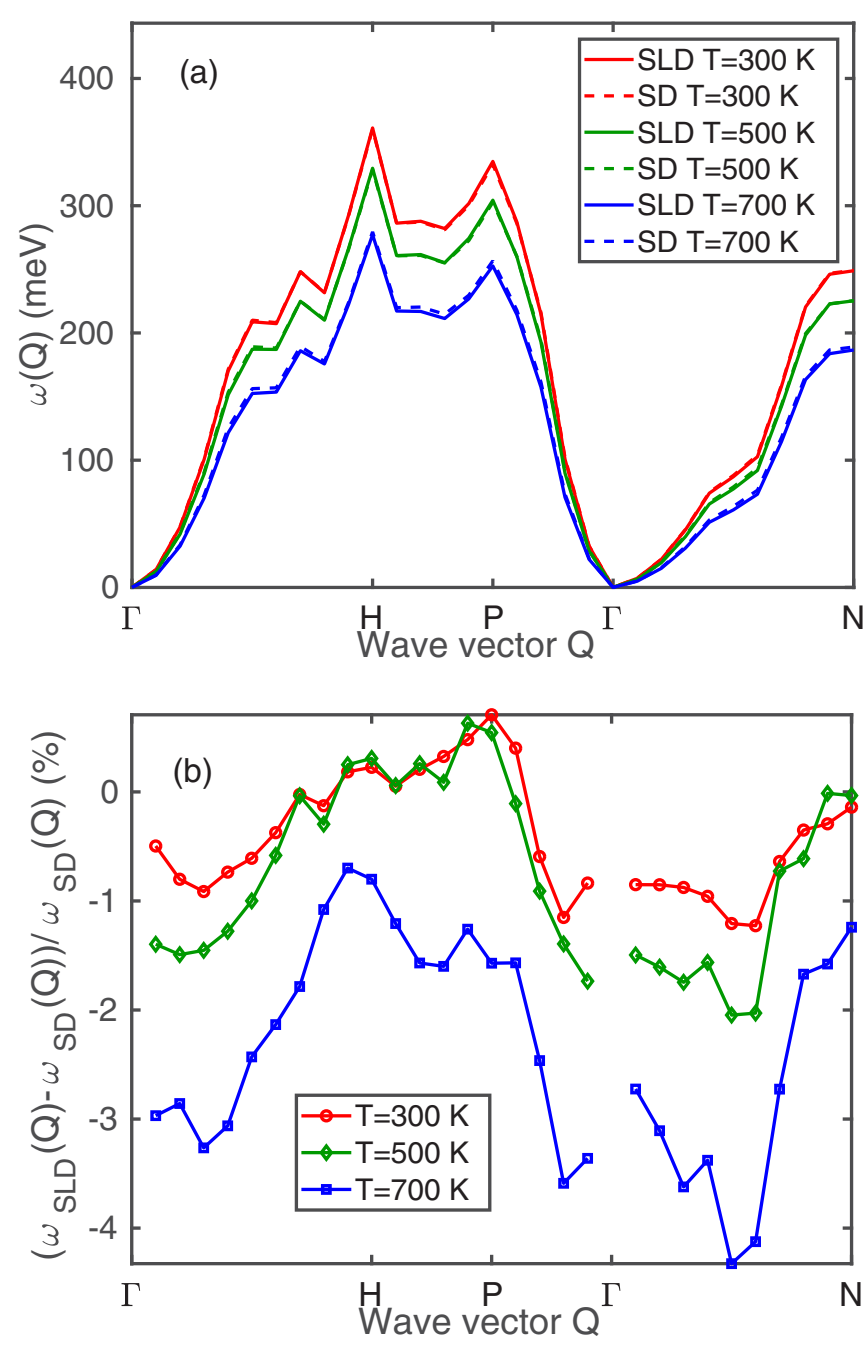

FIG. 17. The dispersions fitted from the spin-spin dynamic structure factor $S_{(\mathbf{m})}(\mathbf{Q}, \omega)$ sampled in SLD simulations and in SD simulations of bcc Fe at different temperatures using a $20 \times 20 \times 20$ supercell with periodic boundary conditions. (a) The finite temperature dispersion $\omega(\mathbf{Q})$ fitted with a Lorentzian function from $S_{(\mathbf{m})}(\mathbf{Q}, \omega)$ SLD (full lines) and SD (dashed lines) simulations at $T=300$ (red), 500 (green), and $700 \mathrm{~K}$ (blue). (b) The ratio $\left(\omega(\mathbf{Q})_{\mathrm{SLD}}-\right.$ $\left.\omega(\mathbf{Q})_{\mathrm{SD}}\right) / \omega(\mathbf{Q})_{\mathrm{SD}}$, at $T=300$ (red), 500 (green), and $700 \mathrm{~K}$ (blue).

largest for intermediate wave vector, and decreasing towards the $H$ point. For the $H-P$ path, there is a slight increase of the dispersion. For both the $P-\Gamma$ and $\Gamma-N$ paths, the magnon soften with up to $2 \%$ for the $T=500 \mathrm{~K}$ simulation.

In concluding this discussion of our simulations for bcc $\mathrm{Fe}$, we note that our results compare overall well with experimental $[21,116,117,119]$ and theoretical $[21,120]$ studies of phonon dispersions at finite temperature, and with reported experimental [121] and theoretical [59,78,95,112] magnon dispersions. Exchange striction was seen to have an impact both on the phonon and the magnon dispersions. When, including Heisenberg exchange interactions up to six lattice constants, we obtained a fine structure for high wave vectors in the magnon dispersions as observed also in earlier ab initio modeling of bcc Fe [112,122]. 


\section{CONCLUSIONS AND OUTLOOK}

In conclusion, we have demonstrated a computationally efficient and general method for performing coupled spinlattice simulations. The method is, in short, based on a Taylor expansion of the bilinear exchange interaction term of the spin-Hamiltonian with respect to nuclear positions, and a Taylor expansion of the force constant tensor with respect to magnetic moment directions. We indicate how the expansion can be carried out in general, to desired order, and that it leads to a coupling term of an effective Hamiltonian for spinlattice dynamics. The methodology presented here has been implemented in the UppASD code [123].

In general, the interaction between the spin and lattice degrees of freedom can be expected to lead to significant changes in both the magnon and phonon spectra of solids, as well as for magnetization dynamics and atomic vibrations of clusters and molecular systems. In our conceptual simulations for small magnetic clusters, we observe new modes emerging as a result of strong interaction between atomic and spin motion. We propose that these coupled modes should be detectable in Raman spectroscopy. In particular, classes of clusters with similar chemical interactions but different magnetic configurations could be compared, and possible different behavior of the vibration frequencies can be identified with spin-lattice coupling. Examples of systems to investigate in this regard would be rare-earth based clusters, either in elemental form or as oxides [124,125]. As examples of suitable systems to investigate we note that $\mathrm{Lu}$ and $\mathrm{La}$ are trivalent elements without a magnetic moment, while, e.g., $\mathrm{Gd}$ and $\mathrm{Tb}$ are trivalent, magnetic materials.

In this work, we also performed simulations of bulk bcc Fe, obtaining good agreement with results of previous modeling of this system. For bcc Fe, the effect of spin-lattice coupling is not dramatic, and it may be difficult to detect experimentally the influence of spin-lattice coupling. In general, both Raman and neutron scattering can be used to detect frequencies of collective modes of materials, but traditionally neutron scattering is a more appropriate tool for these measurements, since it offers the possibility to map out dispersion. To highlight spin-lattice coupling and the influence on collective modes of solids, it would be useful to investigate a material that can be stabilized in a nonmagnetic and a magnetic configuration, where again differences of frequencies of the two phases can be assigned to spin-lattice effects. A possible compound here is the cubic Laves phase of $\mathrm{YCo}_{2}$ that has a well-established metamagnetic phase transition. We also suggest that large effects of spin-lattice coupling may be found in, e.g., Invar (Fe-Ni) alloys, fcc $\mathrm{Fe}$ and materials close to a structural phase transition, where the magnetic moment and/or exchange interaction also changes drastically. This may be found in several compounds that are relevant for magnetocaloric applications.

In simulations that allow for spin-lattice coupling, as presented here, one needs to address if additional mechanisms need to be considered as regards the damping. The reason is that dissipation of energy and angular momentum is allowed directly from the magnetic sub-system into the lattice, for coupled spin-lattice dynamical simulations. This is not the case for pure spin-dynamics simulations. In principle, one would then expect the damping parameter to be lower in coupled spin-lattice dynamics. To bring the mechanism for angular momentum transfer and angular momentum loss onto the same footing, (i) a spin-lattice damping $\alpha^{m l}$ needs to be considered such as in Ref. [11], (ii) striction effects for the magnetic damping, as motivated in Ref. [126], require profound investigation, and (iii) those dissipation quantities needs to be determined from first principles by the KuboGreenwood perturbation methods, as it is done for the Gilbert damping, e.g., in Ref. [127]. Work along the directions outlined in the above outlook is currently being pursued.

\section{ACKNOWLEDGMENTS}

We acknowledge fruitful discussions with Pavel $\mathrm{F}$. Bessarab, Jonas Fransson, Pablo Maldonado, Johan H. Mentink, and Lars Nordström. Financial support from the Swedish Research Council, the Swedish Energy Agency (Energimyndigheten) and the Foundation for Strategic Research (SSF) is acknowledged. J.H. is partly funded by the Swedish Research Council (VR) through a neutron project grant (BIFROST, Dnr. 2016-06955). O.E. also acknowledges the support from eSSENCE, the Knut and Alice Wallenberg (KAW) Foundation, STandUPP. The computations were performed on resources provided by the Swedish National Infrastructure for Computing (SNIC) at the Uppsala Multidisciplinary Center for Advanced Computational Science (UPPMAX), the National Supercomputer Center (NSC), Linköping University, the PDC Centre for High Performance Computing (PDC-HPC), KTH, and the High Performance Computing Center North (HPC2N), Umeå University. We acknowledge fruitful and constructive reviews from the two referees of this paper.

\section{APPENDIX: NUMERICAL INTEGRATION OF THE SPIN-LATTICE DYNAMICS EQUATIONS OF MOTION}

In this Appendix, the schemes we use for numerical integration of the coupled equation of motions expressed in Eqs. (14)-(16) are described. Explicit methods for integrating the stochastic LLG equation are commonly two-step numerical integration as is the case for the Heun method [128], the Depondt-Merten's method [129], and the semi-implicit SIB method by Mentink et al. [72]. A description of these methods, including benchmarks, can be found in Ref. [8]. The Depondt-Merten's method and the semi-implicit SIB method can be extended with a suitable explicit or semi-implicit solver for the lattice degrees of freedom, such as the velocity-Verlet method. Note that integration with Heun or other explicit Runge-Kutta schemes is well known to have poor stability for molecular dynamics.

For the Hamiltonian simulations, we use a fixed-point iteration of the implicit midpoint scheme [130]. This scheme preserves spin strengths and energy. For the simulations in the canonical ensemble, we use a combination of the GrønbechJensen and Farago (G-JF) [73] Verlet-type methods for simulation of Langevin molecular dynamics and the Mentink et al. semi-implicit SIB method for the stochastic LLG equation [72]. This scheme is computationally efficient and preserves spin strengths but not energy. Due to the latter, it was seen 
to not be stable enough for the Hamiltonian simulations. The combined algorithm for the canonical simulation is written out in pseudocode below.

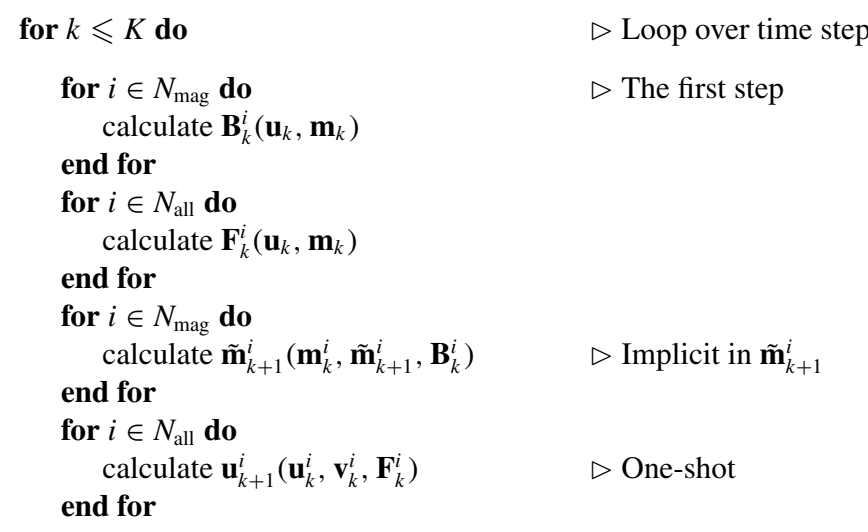

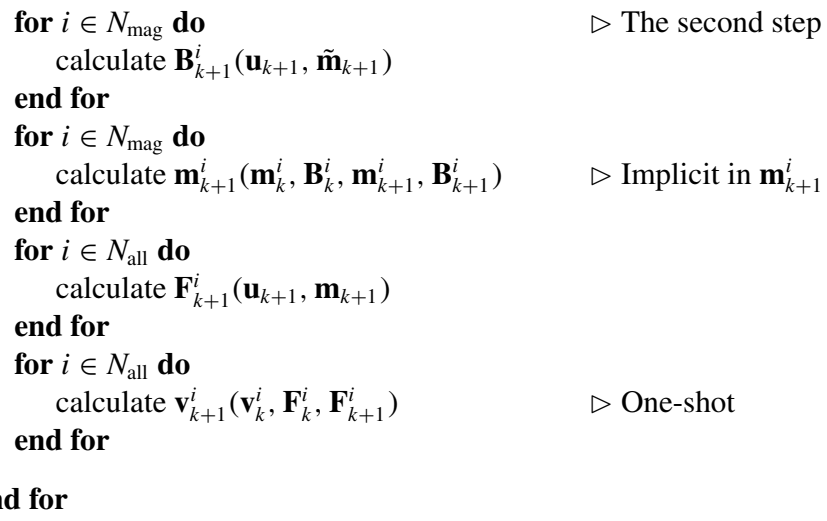

[1] R. Car and M. Parrinello, Phys. Rev. Lett. 55, 2471 (1985).

[2] D. Marx and J. Hutter, Ab Initio Molecular Dynamics: Basic Theory and Advanced Methods (Cambridge University Press, Cambridge, 2009).

[3] J. Fidler and T. Schrefl, J. Phys. D. Appl. Phys. 33, R135 (2000).

[4] V. P. Antropov, M. I. Katsnelson, B. N. Harmon, M. van Schilfgaarde, and D. Kusnezov, Phys. Rev. B 54, 1019 (1996).

[5] U. Nowak, O. Mryasov, R. Wieser, K. Guslienko, and R. Chantrell, Phys. Rev. B 72, 172410 (2005).

[6] B. Skubic, J. Hellsvik, L. Nordström, and O. Eriksson, J. Phys. Condens. Matter 20, 315203 (2008).

[7] R. F. L. Evans, W. J. Fan, P. Chureemart, T. A. Ostler, M. O. A. Ellis, and R. W. Chantrell, J. Phys. Condens. Matter 26, 103202 (2014).

[8] O. Eriksson, A. Bergman, L. Bergqvist, and J. Hellsvik, Atomistic Spin Dynamics (Oxford University Press, 2017), Vol. 1.

[9] M. Katsnelson and V. Antropov, Phys. Rev. B 67, 140406 (2003).

[10] S. Bhattacharjee, L. Nordström, and J. Fransson, Phys. Rev. Lett. 108, 057204 (2012).

[11] J. Fransson, D. Thonig, P. F. Bessarab, S. Bhattacharjee, J. Hellsvik, and L. Nordström, Phys. Rev. Mater. 1, 074404 (2017).

[12] K. Krieger, J. K. Dewhurst, P. Elliott, S. Sharma, and E. K. U. Gross, J. Chem. Theory Comput. 11, 4870 (2015).

[13] P. Maldonado, K. Carva, M. Flammer, and P. M. Oppeneer, Phys. Rev. B 96, 174439 (2017).

[14] I. P. Omelyan, I. M. Mryglod, and R. Folk, Phys. Rev. Lett. 86, 898 (2001).

[15] P.-W. Ma, C. H. Woo, and S. L. Dudarev, Phys. Rev. B 78, 024434 (2008).

[16] M. Shimizu, Reports Prog. Phys. 44, 329 (1981).

[17] R. F. Sabiryanov, S. K. Bose, and O. N. Mryasov, Phys. Rev. B 51, 8958 (1995).

[18] R. F. Sabiryanov and S. S. Jaswal, Phys. Rev. Lett. 83, 2062 (1999).
[19] S. Bonetti, M. C. Hoffmann, M.-J. Sher, Z. Chen, S.-H. Yang, M. G. Samant, S. S. P. Parkin, and H. A. Dürr, Phys. Rev. Lett. 117, 087205 (2016).

[20] H. Hasegawa, M. W. Finnis, and D. G. Pettifor, J. Phys. F Met. Phys. 17, 2049 (1987).

[21] F. Körmann, B. Grabowski, B. Dutta, T. Hickel, L. Mauger, B. Fultz, and J. Neugebauer, Phys. Rev. Lett. 113, 165503 (2014).

[22] E. Carpene, E. Mancini, C. Dallera, M. Brenna, E. Puppin, and S. De Silvestri, Phys. Rev. B 78, 174422 (2008).

[23] M. Wietstruk, A. Melnikov, C. Stamm, T. Kachel, N. Pontius, M. Sultan, C. Gahl, M. Weinelt, H. A. Dürr, and U. Bovensiepen, Phys. Rev. Lett. 106, 127401 (2011).

[24] A. H. Reid, X. Shen, P. Maldonado, T. Chase, E. Jal, P. W. Granitzka, K. Carva, R. K. Li, J. Li, L. Wu, T. Vecchione, T. Liu, Z. Chen, D. J. Higley, N. Hartmann, R. Coffee, J. Wu, G. L. Dakovski, W. F. Schlotter, H. Ohldag, Y. K. Takahashi, V. Mehta, O. Hellwig, A. Fry, Y. Zhu, J. Cao, E. E. Fullerton, J. Stöhr, P. M. Oppeneer, X. J. Wang, and H. A. Dürr, Nat. Commun. 9, 388 (2018).

[25] S. R. Boona and J. P. Heremans, Phys. Rev. B 90, 064421 (2014)

[26] D. J. Sanders and D. Walton, Phys. Rev. B 15, 1489 (1977).

[27] J. W. Kim, M. Vomir, and J. Y. Bigot, Phys. Rev. Lett. 109, 166601 (2012).

[28] A. V. Scherbakov, A. S. Salasyuk, A. V. Akimov, X. Liu, M. Bombeck, C. Brüggemann, D. R. Yakovlev, V. F. Sapega, J. K. Furdyna, and M. Bayer, Phys. Rev. Lett. 105, 117204 (2010).

[29] T. Henighan, M. Trigo, S. Bonetti, P. Granitzka, D. Higley, Z. Chen, M. P. Jiang, R. Kukreja, A. Gray, A. H. Reid, E. Jal, M. C. Hoffmann, M. Kozina, S. Song, M. Chollet, D. Zhu, P. F. Xu, J. Jeong, K. Carva, P. Maldonado, P. M. Oppeneer, M. G. Samant, S. S. P. Parkin, D. A. Reis, and H. A. Dürr, Phys. Rev. B 93, 220301 (2016).

[30] C. M. Jaworski, J. Yang, S. Mack, D. D. Awschalom, R. C. Myers, and J. P. Heremans, Phys. Rev. Lett. 106, 186601 (2011).

[31] P. Man, F. Ma, T. Xie, J. Ding, A. Wu, L. Su, H. Li, and G. Ren, Opt. Mater. (Amst). 66, 207 (2017).

[32] S. F. Maehrlein, I. Radu, P. Maldonado, A. Paarmann, M. Gensch, A. M. Kalashnikova, R. V. Pisarev, M. Wolf, P. M. 
Oppeneer, J. Barker, and T. Kampfrath, Sci. Adv. 4, eaar5164 (2018).

[33] A. Pimenov, A. A. Mukhin, V. Y. Ivanov, V. D. Travkin, A. M. Balbashov, and A. Loidl, Nat. Phys. 2, 97 (2006).

[34] R. Valdés Aguilar, A. B. Sushkov, C. L. Zhang, Y. J. Choi, S.-W. Cheong, and H. D. Drew, Phys. Rev. B 76, 060404(R) (2007).

[35] A. B. Sushkov, M. Mostovoy, R. Valdés Aguilar, S.-W. Cheong, and H. D. Drew, J. Phys. Condens. Matter 20, 434210 (2008).

[36] C. Wang, G.-C. Guo, and L. He, Phys. Rev. B 77, 134113 (2008).

[37] Y. Takahashi, R. Shimano, Y. Kaneko, H. Murakawa, and Y. Tokura, Nat. Phys. 8, 121 (2011).

[38] T. Kubacka, J. A. Johnson, M. C. Hoffmann, C. Vicario, S. de Jong, P. Beaud, S. Grübel, S.-W. Huang, L. Huber, L. Patthey, Y.-D. Chuang, J. J. Turner, G. L. Dakovski, W.-S. Lee, M. P. Minitti, W. Schlotter, R. G. Moore, C. P. Hauri, S. M. Koohpayeh, V. Scagnoli, G. Ingold, S. L. Johnson, and U. Staub, Science 343, 1333 (2014).

[39] D. Wang, J. Weerasinghe, and L. Bellaiche, Phys. Rev. Lett. 109, 067203 (2012).

[40] Y. Tokura, S. Seki, and N. Nagaosa, Rep. Prog. Phys. 77, 076501 (2014).

[41] D. Bossini, K. Konishi, S. Toyoda, T. Arima, J. Yumoto, and M. Kuwata-Gonokami, Nat. Phys. 14, 370 (2018).

[42] A. M. Tishin and Y. I. Spichkin, Int. J. Refrig. 37, 223 (2014).

[43] M. Råsander, L. Bergqvist, and A. Delin, Phys. Rev. B 91, 014303 (2015).

[44] E. Aytan, B. Debnath, F. Kargar, Y. Barlas, M. M. Lacerda, J. X. Li, R. K. Lake, J. Shi, and A. A. Balandin, Appl. Phys. Lett. 111, 252402 (2017).

[45] I. Stockem, A. Bergman, A. Glensk, T. Hickel, F. Körmann, B. Grabowski, J. Neugebauer, and B. Alling, Phys. Rev. Lett. 121, 125902 (2018).

[46] J. Tranchida, S. Plimpton, P. Thibaudeau, and A. Thompson, J. Comput. Phys. 372, 406 (2018).

[47] M. Fechner, A. Sukhov, L. Chotorlishvili, C. Kenel, J. Berakdar, and N. A. Spaldin, Phys. Rev. Mater. 2, 064401 (2018).

[48] G. L. Squires, Introduction to the Theory of Thermal Neutron Scattering (Cambridge University Press, Cambridge, 2012).

[49] E. Beaurepaire, H. Bulou, L. Joly, and F. Scheurer, Magnetism and Synchrotron Radiation: Towards the Fourth Generation Light Sources: Proceedings of the 6th International School "Synchrotron Radiation and Magnetism," Mittelwihr, France, 2012, Springer Proceedings in Physics Vol. 151 (Springer International, Switzerland, 2013).

[50] L. Ament, M. Van Veenendaal, T. Devereaux, J. Hill, and J. Van Den Brink, Rev. Mod. Phys. 83, 705 (2011).

[51] T. Schmitt, F. M. F. de Groot, and J.-E. Rubensson, J. Synchrotron Radiat. 21, 1065 (2014).

[52] J. O. Birk, M. Markó, P. G. Freeman, J. Jacobsen, R. L. Hansen, N. B. Christensen, C. Niedermayer, M. Månsson, H. M. Rønnow, and K. Lefmann, Rev. Sci. Instrum. 85, 113908 (2014).

[53] F. Groitl, D. Graf, J. O. Birk, M. Markó, M. Bartkowiak, U. Filges, C. Niedermayer, C. Rüegg, and H. M. Rønnow, Rev. Sci. Instrum. 87, 035109 (2016).
[54] S. Tóth, B. Wehinger, K. Rolfs, T. Birol, U. Stuhr, H. Takatsu, K. Kimura, T. Kimura, H. M. Rønnow, and C. Rüegg, Nat. Commun. 7, 13547 (2016).

[55] S. L. Dudarev and P. M. Derlet, J. Phys. Condens. Matter 17, 7097 (2005).

[56] P. M. Derlet and S. L. Dudarev, Prog. Mater. Sci. 52, 299 (2007).

[57] P.-W. Ma and S. L. Dudarev, Phys. Rev. B 86, 054416 (2012).

[58] D. Perera, D. P. Landau, D. M. Nicholson, G. Malcolm Stocks, M. Eisenbach, J. Yin, and G. Brown, J. Appl. Phys. 115, 17D124 (2014).

[59] D. Perera, D. M. Nicholson, M. Eisenbach, G. M. Stocks, and D. P. Landau, Phys. Rev. B 95, 014431 (2017).

[60] M. van Schilfgaarde and V. P. Antropov, J. Appl. Phys. 85, 4827 (1999).

[61] J. Lorenzana and G. A. Sawatzky, Phys. Rev. Lett. 74, 1867 (1995).

[62] J. Lorenzana and G. A. Sawatzky, Phys. Rev. B 52, 9576 (1995).

[63] J. Hellsvik, J. H. Mentink, and J. Lorenzana, Phys. Rev. B 94, 144435 (2016).

[64] L. X. Hayden, T. A. Kaplan, and S. D. Mahanti, Phys. Rev. Lett. 105, 047203 (2010).

[65] J. Hellsvik, M. Balestieri, T. Usui, A. Stroppa, A. Bergman, L. Bergqvist, D. Prabhakaran, O. Eriksson, S. Picozzi, T. Kimura, and J. Lorenzana, Phys. Rev. B 90, 014437 (2014).

[66] N. S. Fedorova, C. Ederer, N. A. Spaldin, and A. Scaramucci, Phys. Rev. B 91, 165122 (2015).

[67] M. T. Dove, Introduction to Lattice Dynamics, Cambridge Topics in Mineral Physics and Chemistry (Cambridge University Press, Cambridge, 1993).

[68] A. Szilva, M. Costa, A. Bergman, L. Szunyogh, L. Nordström, and O. Eriksson, Phys. Rev. Lett. 111, 127204 (2013).

[69] W. Zhong, D. Vanderbilt, and K. M. Rabe, Phys. Rev. B 52, 6301 (1995).

[70] A. Kundu and S. Zhang, J. Magn. Magn. Mater. 393, 331 (2015).

[71] P.-W. Ma, S. L. Dudarev, and J. S. Wróbel, Phys. Rev. B 96, 094418 (2017).

[72] J. H. Mentink, M. V. Tretyakov, A. Fasolino, M. I. Katsnelson, and T. Rasing, J. Phys. Condens. Matter 22, 176001 (2010).

[73] N. Grønbech-Jensen and O. Farago, Mol. Phys. 111, 983 (2013).

[74] L. Bergqvist, A. Taroni, A. Bergman, C. Etz, and O. Eriksson, Phys. Rev. B 87, 144401 (2013).

[75] R. Singer, F. Dietermann, D. Steiauf, and M. Fähnle, Phys. Rev. B 76, 052403 (2007).

[76] M. Uhl and J. Kübler, Phys. Rev. Lett. 77, 334 (1996).

[77] N. M. Rosengaard and B. Johansson, Phys. Rev. B 55, 14975 (1997).

[78] S. Halilov, H. Eschrig, A. Perlov, and P. Oppeneer, Phys. Rev. B 58, 293 (1998).

[79] J. Colpa, Phys. A Stat. Mech. Appl. 93, 327 (1978).

[80] S. Toth and B. Lake, J. Phys. Condens. Matter 27, 166002 (2015).

[81] R. Yadav, M. Pereiro, N. A. Bogdanov, S. Nishimoto, A. Bergman, O. Eriksson, J. van den Brink, and L. Hozoi, Phys. Rev. Mater. 2, 380 (2018).

[82] Y. Wang, J. J. Wang, J. E. Saal, S. L. Shang, L.-Q. Chen, and Z.-K. Liu, Phys. Rev. B 82, 172503 (2010). 
[83] S. Baroni and S. de Gironcoli, A. D. Corso, and P. Giannozzi, Rev. Mod. Phys. 73, 515 (2001).

[84] R. M. White, M. Sparks, and I. Ortenburger, Phys. Rev. 139, A450 (1965).

[85] J. H. Kim and J. H. Han, Phys. Rev. B 76, 054431 (2007).

[86] A. Rückriegel, P. Kopietz, D. A. Bozhko, A. A. Serga, and B. Hillebrands, Phys. Rev. B 89, 184413 (2014).

[87] G. Kresse and J. Furthmüller, Phys. Rev. B 54, 11169 (1996).

[88] G. Kresse and J. Furthmüller, Comput. Mater. Sci. 6, 15 (1996).

[89] A. Togo and I. Tanaka, Scr. Mater. 108, 1 (2015).

[90] P. E. Blöchl, Phys. Rev. B 50, 17953 (1994).

[91] G. Kresse and D. Joubert, Phys. Rev. B 59, 1758 (1999).

[92] J. M. Wills, O. Eriksson, P. Andersson, A. Delin, O. Grechnyev, and M. Alouani, Full-Potential Electronic Structure Method, Springer Series in Solid-State Sciences Vol. 167 (Springer Berlin Heidelberg, Berlin, Heidelberg, 2010).

[93] A. I. Liechtenstein, M. I. Katsnelson, and V. A. Gubanov, J. Phys. F Met. Phys. 14, L125 (1984).

[94] A. Liechtenstein, M. Katsnelson, V. Antropov, and V. Gubanov, J. Magn. Magn. Mater. 67, 65 (1987).

[95] Y. O. Kvashnin, O. Grånäs, I. Di Marco, M. I. Katsnelson, a. I. Lichtenstein, and O. Eriksson, Phys. Rev. B 91, 125133 (2015).

[96] S. Paul, D. Iuşan, P. Thunström, Y. O. Kvashnin, J. Hellsvik, M. Pereiro, A. Delin, R. Knut, D. Phuyal, A. Lindblad, O. Karis, B. Sanyal, and O. Eriksson, Phys. Rev. B 97, 125120 (2018).

[97] A. Bergman, J. Hellsvik, P. F. Bessarab, and A. Delin, Sci. Rep. 6, 36872 (2016).

[98] A. Smogunov, A. Dal Corso, A. Delin, R. Weht, and E. Tosatti, Nat. Nanotechnol. 3, 22 (2007).

[99] O. Le Bacq, O. Eriksson, B. Johansson, P. James, and A. Delin, Phys. Rev. B 65, 134430 (2002).

[100] A. Delin and E. Tosatti, Surf. Sci. 566-568, 262 (2004).

[101] J. L. Menéndez, G. Armelles, A. Cebollada, D. Weller, and A. Delin, Phys. Rev. B 62, 10498 (2000).

[102] See Supplemental Material at http://link.aps.org/ supplemental/10.1103/PhysRevB.99.104302 for the movie for the dimer.

[103] J. L. Meriam and L. G. Kraige, Engineering Mechanics, Dynamics, Vol. 2 (Wiley, New York, 1993).

[104] J. Cserti and G. Tichy, Eur. J. Phys. 25, 723 (2004).

[105] See Supplemental Material at http://link.aps.org/ supplemental/10.1103/PhysRevB.99.104302 for the movie for the trimer.

[106] P.-W. Ma, S. L. Dudarev, and C. H. Woo, Phys. Rev. B 85, 184301 (2012).

[107] D. Perera, M. Eisenbach, D. M. Nicholson, G. M. Stocks, and D. P. Landau, Phys. Rev. B 93, 060402 (2016).
[108] G. J. Ackland, M. C. Warren, and S. J. Clark, J. Phys. Condens. Matter 9, 7861 (1997).

[109] D. Finkenstadt, N. Bernstein, J. L. Feldman, M. J. Mehl, and D. A. Papaconstantopoulos, Phys. Rev. B 74, 184118 (2006)

[110] J. W. Flocken and J. R. Hardy, Phys. Rev. 177, 1054 (1969)

[111] W. Frank, C. Elsässer, and M. Fähnle, Phys. Rev. Lett. 74, 1791 (1995).

[112] M. Pajda, J. Kudrnovský, I. Turek, V. Drchal, and P. Bruno, Phys. Rev. B 64, 174402 (2001).

[113] A. Szilva, D. Thonig, P. F. Bessarab, Y. O. Kvashnin, D. C. M. Rodrigues, R. Cardias, M. Pereiro, L. Nordström, A. Bergman, A. B. Klautau, and O. Eriksson, Phys. Rev. B 96, 144413 (2017).

[114] R. Cardias, A. Szilva, A. Bergman, I. D. Marco, M. I. Katsnelson, A. I. Lichtenstein, L. Nordström, A. B. Klautau, O. Eriksson, and Y. O. Kvashnin, Sci. Rep. 7, 4058 (2017).

[115] P.-W. Ma, S. L. Dudarev, A. A. Semenov, and C. H. Woo, Phys. Rev. E 82, 031111 (2010).

[116] V. J. Minkiewicz, G. Shirane, and R. Nathans, Phys. Rev. 162, 528 (1967).

[117] S. Klotz and M. Braden, Phys. Rev. Lett. 85, 3209 (2000).

[118] W. Kohn, Phys. Rev. Lett. 2, 393 (1959).

[119] B. Brockhouse, H. Abou-Helal, and E. Hallman, Solid State Commun. 5, 211 (1967).

[120] M. Müller, P. Erhart, and K. Albe, J. Phys. Condens. Matter 19, 326220 (2007).

[121] J. W. Lynn, Phys. Rev. B 11, 2624 (1975).

[122] S. V. Halilov, A. Y. Perlov, P. M. Oppeneer, and H. Eschrig, Europhys. Lett. 39, 91 (1997).

[123] Uppsala Atomistic Spin Dynamics (UppASD) code Available under GNU General Public License, http://physics.uu.se/ uppasd and http://github.com/UppASD/UppASD.

[124] L. Peters, I. Di Marco, M. S. Litsarev, A. Delin, M. I. Katsnelson, A. Kirilyuk, B. Johansson, B. Sanyal, and O. Eriksson, Phys. Rev. B 92, 035143 (2015).

[125] L. Peters, S. Ghosh, B. Sanyal, C. van Dijk, J. Bowlan, W. de Heer, A. Delin, I. Di Marco, O. Eriksson, M. I. Katsnelson, B. Johansson, and A. Kirilyuk, Sci. Rep. 6, 19676 (2016).

[126] D. Thonig, Y. Kvashnin, O. Eriksson, and M. Pereiro, Phys. Rev. Mater. 2, 013801 (2018).

[127] D. Thonig and J. Henk, New J. Phys. 16, 013032 (2014).

[128] W. Rümelin, SIAM J. Numer. Anal. 19, 604 (1982).

[129] P. Depondt and F. G. Mertens, J. Phys. Condens. Matter 21, 336005 (2009).

[130] U. M. Ascher and L. R. Petzold, Computer Methods for Ordinary Differential Equations and Differential-Algebraic Equations (Society for Industrial and Applied Mathematics, Philadelphia, 1998). 